\title{
Phenotypes of Drosophila Brain Neurons in Primary Culture Reveal a Role for Fascin in Neurite Shape and Trajectory
}

\author{
Robert Kraft, ${ }^{1}$ Mindy M. Escobar, ${ }^{1}$ Martha L. Narro, ${ }^{1}$ Jackie L. Kurtis, ${ }^{1}$ Alon Efrat, ${ }^{2}$ Kobus Barnard,${ }^{2,3}$ and \\ Linda L. Restifo ${ }^{1,3,4}$ \\ ${ }^{1}$ Arizona Research Laboratories Division of Neurobiology, ${ }^{2}$ Department of Computer Science, and ${ }^{3}$ Interdisciplinary Program in Cognitive Science, \\ University of Arizona, Tucson, Arizona 85721, and ${ }^{4}$ Department of Neurology, Arizona Health Sciences Center, Tucson, Arizona 85724
}

\begin{abstract}
Subtle cellular phenotypes in the CNS may evade detection by routine histopathology. Here, we demonstrate the value of primary culture for revealing genetically determined neuronal phenotypes at high resolution. Gamma neurons of Drosophila melanogaster mushroom bodies (MBs) are remodeled during metamorphosis under the control of the steroid hormone 20-hydroxyecdysone (20E). In vitro, wild-type $\gamma$ neurons retain characteristic morphogenetic features, notably a single axon-like dominant primary process and an arbor of short dendrite-like processes, as determined with microtubule-polarity markers. We found three distinct genetically determined phenotypes of cultured neurons from grossly normal brains, suggesting that subtle in vivo attributes are unmasked and amplified in vitro. First, the neurite outgrowth response to $20 \mathrm{E}$ is sexually dimorphic, being much greater in female than in male $\gamma$ neurons. Second, the $\gamma$ neuron-specific "naked runt" phenotype results from transgenic insertion of an MB-specific promoter. Third, the recessive, panneuronal "filagree" phenotype maps to singed, which encodes the actin-bundling protein fascin. Fascin deficiency does not impair the 20E response, but neurites fail to maintain their normal, nearly straight trajectory, instead forming curls and hooks. This is accompanied by abnormally distributed filamentous actin. This is the first demonstration of fascin function in neuronal morphogenesis. Our findings, along with the regulation of human Fascin1 (OMIM 602689) by CREB (cAMP response element-binding protein) binding protein, suggest FSCN1 as a candidate gene for developmental brain disorders. We developed an automated method of computing neurite curvature and classifying neurons based on curvature phenotype. This will facilitate detection of genetic and pharmacological modifiers of neuronal defects resulting from fascin deficiency.
\end{abstract}

Key words: mushroom body; neurogenetics; actin; ecdysone; software; neurite curvature; sexual dimorphism; plasticity; cytoskeleton

\section{Introduction}

Neuronal architecture is critical to proper function of the developing and mature brain. Normal morphology of axonal and dendritic arbors is essential for establishing functional synaptic circuits, and their modification is central to developmental and experience-dependent plasticity (Cohen-Cory, 2002). Accordingly, defective neuronal morphogenesis may be associated with hereditary or acquired neurobehavioral disorders (e.g., mental retardation and drug addiction) (Kaufmann and Moser, 2000; Blanpied and Ehlers, 2004). Anatomical findings are important for understanding the cellular bases of cognitive deficits and

\footnotetext{
Received May 17, 2006; revised July 14, 2006; accepted July 16, 2006.

This work was supported by National Institutes of Health Grants NS028495 (L.L.R.) and T34 GM008718 (M.M.E.). We appreciate the advice and technical support of many colleagues, including C. Wenk (geometric algorithms); M. Inlow (statistics); B. Reddoch, K. Shupe, C. Michel, and A. Pierson (fly food); C. Turner, S. Pickens, and N. Ingraham (cell culture); P. Jansma (microscopy); T. Yuhas (computers); and M. Aliff (data analysis). S. Sanyal and C. Cherry originally suggested the use of Nod and Kinesin markers in vitro. We thank M. Ellisman (University of California, San Diego, La Jolla, (A) for suggesting that fascin might be involved in the filagree phenotype and Village Silversmith Gallery (Bellport, NY) for permission to show their filagree earrings.

Correspondence should be addressed to Linda L. Restifo, University of Arizona, 1040 East 4th Street, Tucson, AZ 85721-0077. E-mail: LLR@neurobio.arizona.edu.

M. M. Escobar's present address: Program in Neuroscience, University of Arizona, LSN 351, Tucson, AZ 85724.

J. L. Kurtis's present address: Arizona State University College of Law, P.0. Box 877906, Tempe, AZ 85287-7906.

DOI:10.1523/JNEUROSCI.2106-06.2006

Copyright $\odot 2006$ Society for Neuroscience $\quad$ 0270-6474/06/268734-14\$15.00/0
}

could promote bioassay development for therapeutic drug discovery. However, they require animal models or postmortem brain tissue, which reveals the terminal phenotype but not necessarily its pathogenesis. Furthermore, sophisticated methods are necessary to detect subtle defects (e.g., abnormal dendritic spines), increasing the likelihood that they go unrecognized. Hence, in vitro approaches play important roles in revealing cellular phenotypes.

In vitro studies of neuron-morphogenesis defects caused by drugs of abuse, for example, complement in vivo analyses of brain development and behavior (Nassogne et al., 1995; Lindsley et al., 2003; Robinson and Kolb, 2004). In the realm of neurogenetic disease, cell-culture models of spinal muscular atrophy suggest roles for the causative gene in neurite outgrowth and axonal transport that were not apparent from spinal cord histopathology (Briese et al., 2005). Thus, a progressive disease that is routinely classified as neurodegenerative (Victor and Ropper, 2001) may have a developmental component to its pathogenesis.

Here, we report the use of primary cell culture in Drosophila to reveal unexpected neuronal phenotypes and identify a candidate gene for developmental brain disorders. We were specifically motivated by studies demonstrating defective growth cone motility (Kim and Wu, 1996) and electrophysiology (Rohrbough et al., 2003) of brain neurons from learning or memory mutants. More 
generally, because of phylogenetic conservation, the Drosophila genetic model system shows great promise in the study of neurological disorders (Bier, 2005), particularly mental retardation (Inlow and Restifo, 2004; Restifo, 2005).

To detect morphogenetic phenotypes, a neuronal culture system must allow high-resolution imaging of neurite arbors. We use immunofluorescent imaging of neuronal membranes for quantitative analysis (Kraft et al., 1998; our study). Sensitivity is further enhanced by methods that mark neuronal subsets (Boquet et al., 2000), such as those for identification of cultured neurons from the mushroom bodies (Kraft et al., 1998; Rohrbough et al., 2003), an insect brain region involved in cognition (Heisenberg, 2003). The neurons so identified should have a characteristic wild-type morphology (e.g., with distinct axonal and dendritic arbors), which we demonstrate below. Ideally, the cultured neurons should manifest developmentally relevant plasticity. During metamorphosis, Drosophila mushroom body (MB) $\gamma$ neurons undergo steroid hormone-dependent remodeling in vivo (Lee et al., 1999). In vitro, they respond to the molting hormone with enhanced neurite outgrowth (Kraft et al., 1998), through a mechanism we address here. Using primary cultured neurons from developing Drosophila CNS, we characterized three distinct phenotypes: sexual dimorphism among wild-type MB $\gamma$ neurons, an MB-selective defect of neurite outgrowth, and a pan-neuronal alteration of neurite morphogenesis that confirms a long-suspected role for fascin.

\section{Materials and Methods}

Drosophila rearing and genetics. Stocks were maintained at room temperature on corn meal food from the Tucson Drosophila Species Stock Center (http://stockcenter.arl.arizona.edu/index.php?option $=\mathrm{com}_{-}$ content\&task $=$ view\&id $=23 \& I t e m i d=37)$. Experimental cultures were reared as described previously (Kraft et al., 1998) with supplemental dry active yeast (Fleischman's) at $25^{\circ} \mathrm{C}$ and $60-80 \%$ relative humidity. Control strains were OreRC and the " $w(z)$ " stock of $w^{1118}$ in a Canton-S background, the latter obtained from T. Tully (Cold Spring Harbor Laboratory, Cold Spring Harbor, NY). We used the GAL4/UAS system to drive selective expression of reporter genes (Brand and Dormand, 1995). The $P[$ Gal4]201Y enhancer detector was used to mark MB $\gamma$ neurons during larval and pupal stages (Kraft et al., 1998). Stocks carrying reporter-gene markers of axons ( $y w$; UAS-Khc-lacZ/TM3, Sb) or dendrites ( $y$ w; UAS-Nod-lacZ/TM3, Sb) (Clark et al., 1997) were provided by $\mathrm{S}$. Younger and Y. N. Jan (University of California at San Francisco, San Francisco, CA) and by C. Cherry and R. Levine (University of Arizona, Tucson, AZ), respectively. Stocks of a recessive lethal mutation of $E 74 A$, E74A ${ }^{\text {Pneo }}$ mwh red $e^{s} / \mathrm{TM} 6 \mathrm{C}, \mathrm{Tb} \mathrm{Sb}$ e cu ca and a deficiency chromosome that uncovers E74, Df(3L) st-81 ${ }^{k 19} / \mathrm{TM} 6 \mathrm{C}, \mathrm{Sb}$ Tb (Fletcher et al., 1995) were obtained from C. Thummel (University of Utah, Salt Lake City, UT). The dMEF2-lacZ transgene (Schulz et al., 1996) was provided by R. A. Schulz (University of Texas, Houston, TX) as homozygous secondand third-chromosome stocks. A variety of Binsn- or FM7i-GFPbalanced Broad Complex mutations previously used to demonstrate nervous system phenotypes (Restifo and White, 1991) were used in the initial analysis and mapping of the filagree phenotype. These included $y$ $r b p^{5} w^{a} s n^{3} / B i n s n, y n p r 1^{3} w s n^{3} / B i n s n, y r b p^{5} w^{a} s n^{3} / y n p r 1^{3} w s n^{3}$, as well as Binsn/Binsn, Binsn/Y, and a laboratory stock of $y w s n^{3}$. Stocks of two singed alleles, the hypomorph $s n^{3}$ (homozygous) and the probable null $s n^{X 2}\left[\operatorname{In}(1) M u d, M u d^{1} / \operatorname{In}(1) d l-49, s n^{X 2}\right]$ were obtained from G. Guild (University of Pennsylvania, Philadelphia, PA).

Standard matings and recombination methods were performed as needed. Animals were selected at readily identifiable developmental stages. For whole-mount immunostaining or primary cell culture, the CNS was microdissected from wandering third instar larvae, pupae at head eversion (HE), pupae at $5 \mathrm{~h}$ after head eversion $(\mathrm{HE}+5 \mathrm{~h})$, or pharate adults $<12 \mathrm{~h}$ before eclosion. For the pupal samples, animals were collected as young prepupae, sexed, maintained in a humid cham- ber at $25^{\circ} \mathrm{C}$, and monitored at $15 \mathrm{~min}$ intervals for $\mathrm{HE}$, indicating the onset of pupal development. $\mathrm{HE}+5 \mathrm{~h}$ is just before the onset of the large pupal peak of ecdysteroids (Bainbridge and Bownes, 1988). We confirmed the survival of approximately half of $E 74 A^{\text {Pneo }} / D f(3 L) s t-81^{k 19} \mathrm{mu}-$ tants to early pupal stages and selected only healthy animals for experiments.

Primary neuronal cell cultures. Dissociated neuronal cultures from Drosophila brain tissue were prepared as described previously (Kraft et al., 1998) with minor modifications. The CNS was dissected in culture medium [final concentration, $0.88 \times$ Schneider's Insect Medium (Invitrogen, San Diego, CA), 10\% fetal bovine serum (Hyclone, Logan, UT), $50 \mu \mathrm{g} / \mathrm{ml}$ insulin (Sigma, St. Louis, MO)], and the central brain region was isolated by removal of optic lobes and thoracoabdominal ganglion. Cellular dissociation was accomplished by a combination of enzymatic digestion with collagenase and dispase (Worthington and Sigma, respectively, or Roche Applied Science, Indianapolis, IN) and mechanical trituration by pipetting. One hundred microliter aliquots of the cell suspension, representing one-quarter or one-sixth of the total brain sample, were dispensed into 8-mm-diameter wells with floors made from gridded glass coverslips (Bellco, Vineland, NJ) coated with Conconavalin A (Sigma) and laminin (BD Biosciences, Franklin Lakes, NJ). After a 2-3 h incubation at $25^{\circ} \mathrm{C}$ to allow the cells to adhere to the substrate, the dishes were flooded with an additional $900 \mu \mathrm{l}$ of culture medium. The insect steroid hormone 20-hydroxyecdysone (20E) (Sigma) was added at a final concentration of $1.0 \mu \mathrm{g} / \mathrm{ml}\left(2.1 \times 10^{-6} \mathrm{M}\right)$. Neurons were cultured for $1-3 \mathrm{~d}$ at $25^{\circ} \mathrm{C}$.

Immunostaining and microscopy. Fixation and other general immunohistochemical methods were performed as described previously (Kraft et al., 1998). Figures were assembled and labeled using Corel PhotoPaint v. 9 and CorelDraw v. 9 (Corel, Ottawa, Ontario, Canada).

Mushroom body morphology in whole-mount preparations was revealed by using a preabsorbed polyclonal rabbit anti- $\beta$-galactosidase ( $\beta$ gal) antiserum (Cappel, West Chester, PA) at 1:5000, detected with either an Alexa Fluor 488-conjugated goat anti-rabbit antiserum (Invitrogen) at 1:500 or a Cy3-conjugated goat anti-rabbit antiserum (Jackson ImmunoResearch, West Grove, PA) at 1:250. Fluorescently stained tissue was imaged by laser-scanning confocal microscopy using a Nikon (Tokyo, Japan) PCM 2000 mounted on a Nikon E800 upright microscope, with either $40 \times$ (numerical aperture, 1.0) or $60 \times$ (numerical aperture, 1.4) objectives and SimplePCI image acquisition software (Compix, version 4.0.6). A helium-neon laser, with an excitation maximum at $543 \mathrm{~nm}$ and a long-pass filter at $565 \mathrm{~nm}$ was used to detect Cy3 or rhodamine. An argon laser, with an excitation maximum at $488 \mathrm{~nm}$ and a bandpass filter at $500-530 \mathrm{~nm}$, was used to detect Alexa Fluor 488 or BODIPY FL.

For staining cultured neurons, neuronal membranes were visualized with a polyclonal goat anti-horseradish peroxidase (anti-HRP) antiserum (Sigma) at 1:500 and detected with an Alexa Fluor 488-conjugated donkey anti-goat antiserum (Invitrogen) at 1:500. When F-actin was visualized along with anti-HRP immunostaining, a Rhodamine Red $\mathrm{X}$-conjugated donkey anti-goat antiserum (Jackson ImmunoResearch) was used at 1:400 along with BODIPY FL phallicidin (Invitrogen) at 1:40. To visualize $\beta$-galactosidase reporter-gene product, a preabsorbed polyclonal rabbit anti- $\beta$ gal antiserum (Cappel) was used at 1:5000 and detected with a Cy3-conjugated donkey anti-rabbit antiserum (Jackson ImmunoResearch) at 1:500. Cultured neurons were examined on a Diaphot 300 inverted microscope (Nikon) with a $60 \times$ oil-immersion objective (numerical aperture, 1.4), using phase-contrast optics for live cells and epifluorescence illumination for labeled cells. Detection of fluorescent signal was with filter cube Chroma \#41001 (exciter 460-500 nm; dichroic $505 \mathrm{~nm}$; bandpass emitter, $510-560 \mathrm{~nm}$ ) for Alexa Fluor 488 and with Nikon G-2A filter cube (exciter, $510-560 \mathrm{~nm}$; dichroic, $580 \mathrm{~nm}$; long-pass emitter, $590 \mathrm{~nm}$ ) for Cy3 or Rhodamine Red X. Images were collected with an N6000 single lens reflex camera (Nikon) and Kodak (Rochester, NY) TMAX 400 Professional Film, a Spot RT digital camera (Diagnostic Instruments, Sterling Heights, MI), or a Hamamatsu ORCA285 digital camera (Hamamatsu Photonic Systems, Bridgewater, NJ). Photographic negatives were scanned at 600 dpi with either an HP ScanJet 3c (Hewlett Packard, Palo Alto, CA) or UMAX Magic Scan 
(UMAX Technologies, Dallas, TX) to generate eight-bit digital images in TIFF format. The digital images were saved as eight- or 16-bit TIFF files. Cells stained with phallicidin to detect F-actin were visualized by laserscanning confocal microscopy (see above), using $60 \times$ (numerical aperture, 1.4) or $100 \times$ (numerical aperture, 1.4) objectives.

Cultured-neuron image collection and analysis. MB $\gamma$ neurons (i.e., those with 201Y-directed reporter gene expression) were identified by fluorescence microscopy. Alphanumeric grids on the floors of the culture dishes facilitated efficient and systematic selection of $\beta$ gal-expressing neurons. For quantitative morphometric analysis, the dish was scanned starting at the left side of the field and progressing up and down along successively rightward columns of the grid. The anti-HRP signal of every $\beta \mathrm{gal}(+)$ neuron was imaged, unless it was extensively entangled with a neighbor, until at least 50 neurons were collected. On rare occasions, subsequent analysis revealed that a neuron image could not be accurately skeletonized because of the interaction with neurites from neighboring cells, and it was eliminated from the sample. For analysis of generic brain neurons, one or two $\beta$ gal $(-)$ neurons in the immediate vicinity of each $\beta \operatorname{gal}(+)$ neuron were imaged. For the evaluation of Nod: $\beta$ gal- and Khc: $\beta$ gal-expressing $\gamma$ neurons, the dish was scanned in the same manner, but neurons making significant contact with neighbors were not imaged. When sampling unmarked central brain neurons for later scoring as "filagree" or "normal," every well-isolated cell overlying every other horizontal gridline was imaged, and the entire dish was sampled.

Using SimplePCI image-analysis software (Compix, version 5), each image was smoothed and, following Laplacian transformation to improve detection of fine neurites and termini with faint signals, skeletonized to generate a one-pixel-wide representation of the neuron. Manual editing was performed to remove the cell body and to correct errors caused by branch crossing, self-fasciculation, interfering neurites from adjacent cells, debris on the dish surface, and background fluorescence. The final edited skeleton represents the complete arbor of neurite processes. Total neurite length in micrometers was computed from the final skeleton using the SimplePCI "Length" measurement function and an appropriate calibration scale. The length of each primary process and its arbor was also measured for determining the Polarity Index (PI) (the percentage of total length contributed by the primary process with the greatest combined length of its main trunk and arbor). A branch is defined as a neuritic process that starts at either the cell body or a branch point and ends at a terminus, not at another branch point. The branch points ("nodes") were identified using the SimplePCI "Break Node" function and, if necessary, manual editing was again performed. The node count plus the number of primary processes equals the total number of branches for the neuron. To determine the territory of each neuron (defined as the area occupied by the footprint of the cell body and arbor), a convex hull was drawn manually around each neuron skeleton and cell body. In other words, a polygon was drawn using the minimal number of segments to connect branch tips (and the cell body if it was located eccentrically) such that all external polygon angles were $>180^{\circ}$. The area of the polygon ("territory") was computed by the SimplePCI "Area" function. Branch-point density was calculated by dividing the total number of branches by the territory.

The nonparametric Mann-Whitney rank sum test was used to compare measures of neuron size and shape (total neurite length, branch number, territory, and branch-point density) between populations, because most data sets were not normally distributed. SigmaStat v. 1.0 software (SYSTAT) was used for these tests and for calculating descriptive statistics, including medians and means. Graphs depicting the data were generated with SigmaPlot 2000 (SYSTAT) or Microsoft Excel 2000 (Microsoft, Seattle, WA).

The use of computer-assisted morphometric assessment of neuronal size and shape represents a considerable improvement over our previous methods based on manual tracing of photographic images. As detailed in the study by Kraft et al. (1998, their Fig. 5), we were unable to include small terminal neurites in either length or branch count because of fixed, per-branch errors inherent in the tracing method. Hence, the morphometric values obtained by our current methods are more complete and accurate, representing the entire neurite arbor, and cannot be directly compared with the older values, which are considerably smaller. Both methods demonstrate that the neurite-outgrowth-promoting effect of $20 \mathrm{E}$ does not change length-to-branch ratios of MB $\gamma$ neurons (Kraft et al., 1998) (data not shown).

Quantifying curvature. An automated method was developed to quantify overall neuron curvature from skeletonized images. Source code for the computational methods will be made available upon request. The general strategy was to fit a cubic curve to the neurite segment containing each point and calculate the curvature at that point as defined by the change in tangent angle relative to the distance traveled along the curve. This point-curvature estimation method for neurites is similar to that developed by others for bristle curvature (Tilney et al., 2004), except that they used degree-four polynomials through selected points. Using degree-three polynomials (cubics) through all available points results in a more easily automated, robust, and parsimonious global fit. The structure of neurite arbors requires that our method determine which path to follow at branch points. The skeletonized images were preprocessed to separate side branches from parent branches (see supplemental information, available at www.jneurosci.org as supplemental material). This resulted in a fragmented, one-pixel-wide skeleton, with unambiguous path selection, that was used for curvature measurements.

Each internal point of a skeletonized neurite fragment has two neighbors, with neighborhood defined as adjacent in eight directions. The segment for each point in question was found by traversing the skeleton in each of the two available directions in turn, stopping when either the path terminated or 16 pixels had been traversed. For the images used in the curvature analysis, $1 \mu \mathrm{m}=4.5$ pixels. Only segments that had at least eight pixels in each direction were considered sufficiently long to yield reliable curvature values. The latter restriction had the effect of eliminating small $(<3.8 \mu \mathrm{m})$ terminal branches from the analysis.

The image points of a curve segment, $x(t)$ and $y(t)$ were represented by $x(t)=a_{x} t^{3}+b_{x} t^{2}+c_{x} t+d_{x}$ and $y(t)=a_{y} t^{3}+b_{y} t^{2}+c_{y} t+d_{y}$. The value of the parameter, $t$, for a given point was approximated by the directed distance traveled along the curve from the point under consideration, going through the pixel centers. In other words, the arc length of the curve section from an arbitrary anchor point was estimated by cumulative steps of either one unit (for up/down, and left/right) or $\sqrt{2}$ (for diagonal moves). Given this approximation, the eight coefficients $\left(a_{x}, b_{x}\right.$, $c_{x}, d_{x}, a_{y}, b_{y}, c_{y}, d_{y}$ ) can be determined in the least-squares sense by forming a matrix, $\mathrm{A}$, whose rows are $\left(t^{3}, t^{2}, t, 1\right)$ for each point, and vectors $\mathbf{x}$ and $\mathbf{y}$ for the $\mathbf{x}$ and $\mathrm{y}$ coordinates of each point. The following was used to solve for $\left(a_{\mathrm{x}}, b_{\mathrm{x}}, c_{\mathrm{x}}, d_{\mathrm{x}}\right)$ and $\left(a_{\mathrm{y}}, b_{\mathrm{y}}, c_{\mathrm{y}}, d_{\mathrm{y}}\right)$ independently in the least-squares sense: $\left(a_{x}, b_{x}, c_{x}, d_{x}\right)^{T}=\mathrm{A}^{\dagger} \mathbf{x}$ and $\left(a_{y}, b_{y}, c_{y}, d_{y}\right)^{T}=\mathrm{A}^{\dagger} \mathbf{y}$, where $\mathrm{A}^{\dagger}$ denotes the Moore-Penrose pseudo-inverse of A. The curve fit obtained with this approach was excellent (see examples in Fig. 9A-D), verifying the adequacy of the best-cubic-curve approximation for this purpose. Given the above parametric cubic, curvature, $\kappa$, can be evaluated using the standard formula, evaluated at $t=0$ by the following:

$$
\kappa=\frac{\dot{x} \ddot{y}-\ddot{x} \dot{y}}{\left(\dot{x}^{2}+\dot{y}^{2}\right)^{3 / 2}}=2 \cdot \frac{c_{\mathrm{x}} b_{\mathrm{y}}-c_{\mathrm{y}} b_{\mathrm{x}}}{\left(c_{\mathrm{x}}^{2}+c_{\mathrm{y}}^{2}\right)^{3 / 2}} .
$$

The absolute value of the curvature, $|\kappa|$ was used. Thus, the method did not require knowledge of the orientation of processes relative to the cell body.

To estimate the overall curvature of an entire neuron, all of the values for $|\kappa|$ were averaged over the usable points in the image, weighted by an estimate of the fractional curve length represented by that pixel, which varied between one (for a horizontal or vertical section) and $\sqrt{2}$ (for a fully diagonal section). Frequency distributions of curvature values for each population were plotted with soft binning, where each data point contributed as a normal distribution with $\sigma$ set to the bin size. A normal distribution using the sample mean and variance was plotted over the data for each group. Comparisons between groups were made using Welch's $t$ test (Myers and Well, 1991), which accounts for different variances and sample sizes.

Classification of neurons based on overall curvature. The differences in the populations were further tested using the model of two groups with normally distributed mean absolute curvature as the basis for classification. Each neuron to be classified was put into the most likely group, 
"normal" or "filagree," based on a decision boundary computed under the assumption of normality. The efficacy of classification was estimated using leave-out-one cross-validation (Hastie et al., 2001). Specifically, each neuron image was removed from the training process, the means and variances were computed for the distributions using the remaining neurons, and then the held-out neuron was classified based on the assumption that the two normal distributions represented the statistics of the data.

\section{Results \\ Response of $\mathrm{MB} \gamma$ neurons to $20 \mathrm{E}$ in vitro depends on the ecdsyone cascade gene $E 74 A$ and sex}

In previous studies of $\mathrm{MB}$ metamorphosis, we demonstrated that genetically marked MB Kenyon cells (the intrinsic neurons) could be identified in dissociated cultures prepared from developing Drosophila brains (Kraft et al., 1998). We used the $P$ [Gal4]201Y enhancer detector ("201Y"), which drives expression of reporter genes in the MB $\gamma$ neurons (" $\gamma$ neurons") during all stages from late larva to adult (Yang et al., 1995; Kraft et al., 1998). During metamorphosis, these neurons undergo axonal and dendritic remodeling (Lee et al., 1999) that is dependent on the ecdysone receptor (EcR) and its dimeric partner, USP (Lee et al., 2000a). In vitro, $201 Y$-labeled early-pupal $\gamma$ neurons express $\mathrm{EcR}$ and respond to $20 \mathrm{E}$ by enhancing their neurite outgrowth in a proportionate manner (i.e., their length:branch ratios are unchanged by the hormone) (Kraft et al., 1998). This suggested that the large surge of ecdysteroids in mid-pupal development (Richards, 1981) promotes the regeneration phase of MB remodeling to ensure complete axonal and dendritic extension in a timely manner.

The high-intensity, high-contrast signal of anti-HRP immunostaining provides an accurate, detailed representation of neurite arbors (Kraft et al., 1998) and allowed a switch from traditional photomicrographs to digital images that can be skeletonized (Fig. 1 $A, B$ ). Using commercial software and manual editing, we represented each neuron image as a single-pixel-wide skeleton (Fig. 1B), from which we extracted morphometric information, including length, branch number, and territory (area occupied by the footprint of cell body and arbor). This method is more accurate for neuron size and shape quantitation than our previous technique (see Materials and Methods for details) and confirmed the in vitro response of $\gamma$ neurons to $20 \mathrm{E}$ (Fig. 1C).

To investigate the molecular pathway underlying the $20 \mathrm{E}$ response of MB $\gamma$ neurons, we studied mutants of the ecdysone cascade primary-response gene, E74A. E74 encodes a pair of 20Einducible transcription factors essential for events throughout metamorphosis (Thummel, 1996). We used a severe genotype $\left[E 74 A^{\text {Pneo }} / D f(3 L) s t-81^{k 19}\right]$ that allows approximately half the mutants to survive to at least early pupal stages (Fletcher et al., 1995). Based on $\gamma$ axon degeneration in the dorsal and medial MB lobes, the mutant neurons undergo the first phase of remodeling normally (data not shown). When cultured in vitro, female E74A mutant pupal $\gamma$ neurons have a greatly diminished response to $20 \mathrm{E}$ compared with that of wild-type neurons, with no statistically significant difference in total length between mutant treatment groups (Fig. 1C). The size range of the mutant neurons was also notably broader than normal, especially in the (-)20E group, which suggests that $E 74 \mathrm{~A}$ mutant neurons may have defective outgrowth regulation in addition to a blunted hormone response.

Our previous studies hinted that male and female MB $\gamma$ neurons have different degrees of $20 \mathrm{E}$ responsiveness (Kraft et al., 1998). To test this hypothesis, we conducted three independent experiments comparing the 20E-response properties of $\gamma$ neurons from pairs of male-female siblings. As in all such experi- ments, each brain served as its own control in that the dissociated neurons were split into several separate dishes for culture in either the presence or absence of 20E. For each pair of siblings, the female neurons showed a large, highly statistically significant increase in total neurite length $(p<0.0001)$ and total branch number $(p<0.0001)$ (Fig. 2). In contrast, the neurons from the male siblings' brains showed no significant change in branch number resulting from hormone treatment (Fig. 2). For total neurite length, the male neurons showed either some increase $(p<$ 0.004; one experiment) or, in two experiments, no statistically significant difference ( $p=0.272$ and $p=0.075)$. No gross morphological differences between mushroom bodies in males and females have been reported at any developmental stage. Nonetheless, a quantitative ultrastructural study found that adult females have larger numbers of Kenyon cell axons than do males (Technau, 1984). Thus, the sexual dimorphism in the $20 \mathrm{E}$ responsiveness of $\gamma$ neurons during metamorphosis may contribute to this difference in the adult.

\section{MB $\gamma$ neuron polarity in vitro results from distinct axon and dendrite arbors}

MB $\gamma$ neurons harvested early in pupal development have a characteristic polar morphology when cultured in vitro. Typically, their cell bodies extend a single major primary neurite, with an extensive arbor of higher-order branches, as well as several short primary processes of variable size and complexity (Fig. 1A). Some neurons are monopolar or stellate. Neuronal polarity can be quantified as the Polarity Index, the percentage of total length contributed by the dominant primary process (defined as the primary process with the greatest combined length of its main trunk and arbor). For $\gamma$ neurons, the population distribution of PI values is highly skewed: the majority of neurons have PI values $>80$, whether or not 20E is present in the culture medium (Kraft et al., 1998). To investigate whether this distinctive polarity is a general feature of all cultured pupal brain neurons or specific to $\gamma$ neurons, we analyzed the PI profiles of $201 Y$-positive cells and their adjacent 201Y-negative neighbors ("generic brain neurons") after $3 \mathrm{~d}$ in vitro (div) with $20 \mathrm{E}$ (Fig. $3 A$ ). In contrast to the marked skew of the $\gamma$-neuron PI profile, generic brain neurons have lower PI values, with a suggestion of greater heterogeneity. Whereas $58 \%$ of the $\gamma$ neurons had a PI $\geq 80$, only $25 \%$ of the generic neurons displayed this high degree of polarity. Conversely, $16 \%$ of the $\gamma$ neurons had a PI $\leq 60$, whereas $56 \%$ of the generic neurons exhibited low polarity. PI values of 40-60 indicate a stellate morphology (Kraft et al., 1998). In summary, pupal $\gamma$ neurons extend arbors with a characteristic polar morphology that distinguishes them from their culture-dish neighbors derived from other brain regions.

We noted previously (Kraft et al., 1998) that the typical in vitro morphology of $\mathrm{MB} \gamma$ neurons resembles their in vivo appearance and suggests an endogenous morphogenetic program like that shown for hippocampal pyramidal neurons (Esch et al., 2000; Jacobson et al., 2006). In particular, the dominant primary process is reminiscent of the $\gamma$ neuron axon, and the short primary processes resemble dendrites. We used in vivo markers of Drosophila axons and dendrites, based on microtubule orientation differences (Clark et al., 1997), to test this hypothesis. Microtubules in axons have a uniform orientation, with the plus ends directed away from the cell body, whereas in dendrites they are heterogeneous, with both plus and minus ends directed away from the cell body (Baas, 1999). These differences can be exploited to distinguish the two types of neuronal processes, because distinct motor proteins are directed to the plus and minus 


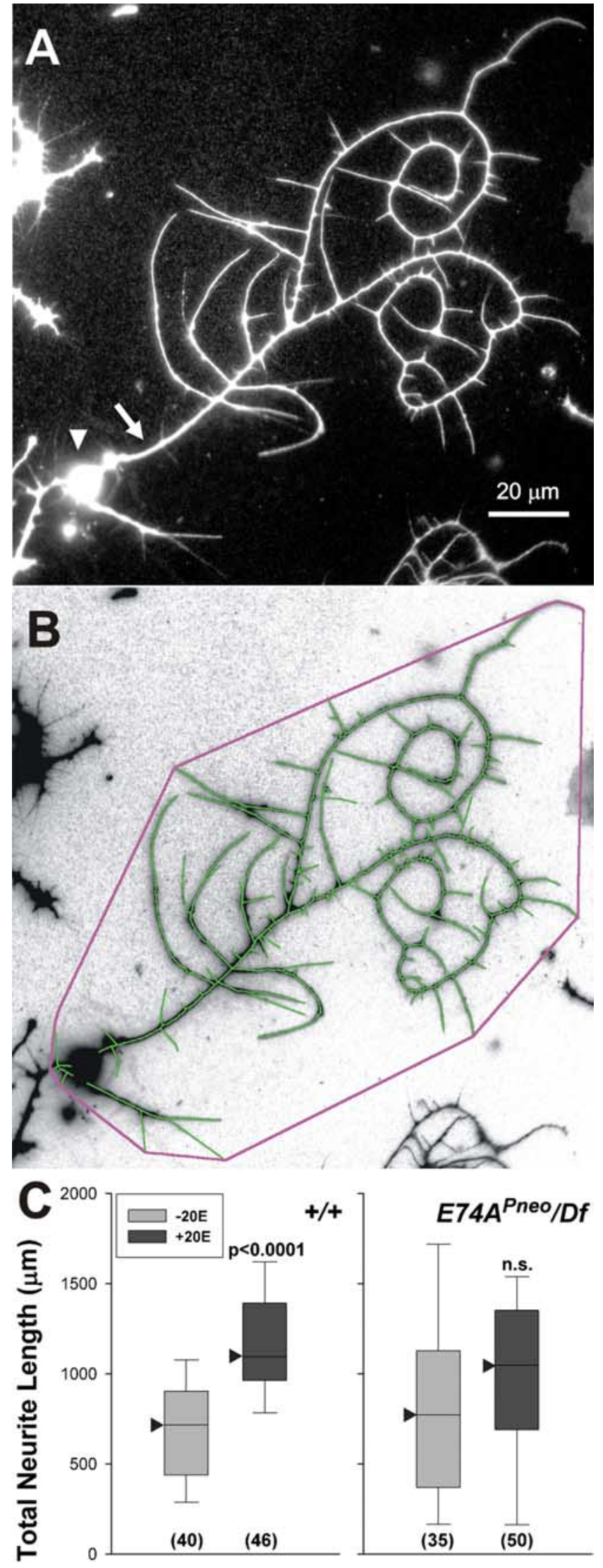

Figure 1. Computer-assisted analysis of MB $\gamma$ neurons demonstrates E74A-dependent neurite-outgrowth response to 20E. $A$, Wild-type $201 Y$-marked $\gamma$ neuron, from a pupal brain dissociated at $\mathrm{HE}+5 \mathrm{~h}$ and cultured 3 div, visualized by immunofluorescent staining of neuronal membranes with anti-HRP. Triangle, cell body; arrow, proximal portion of dominant

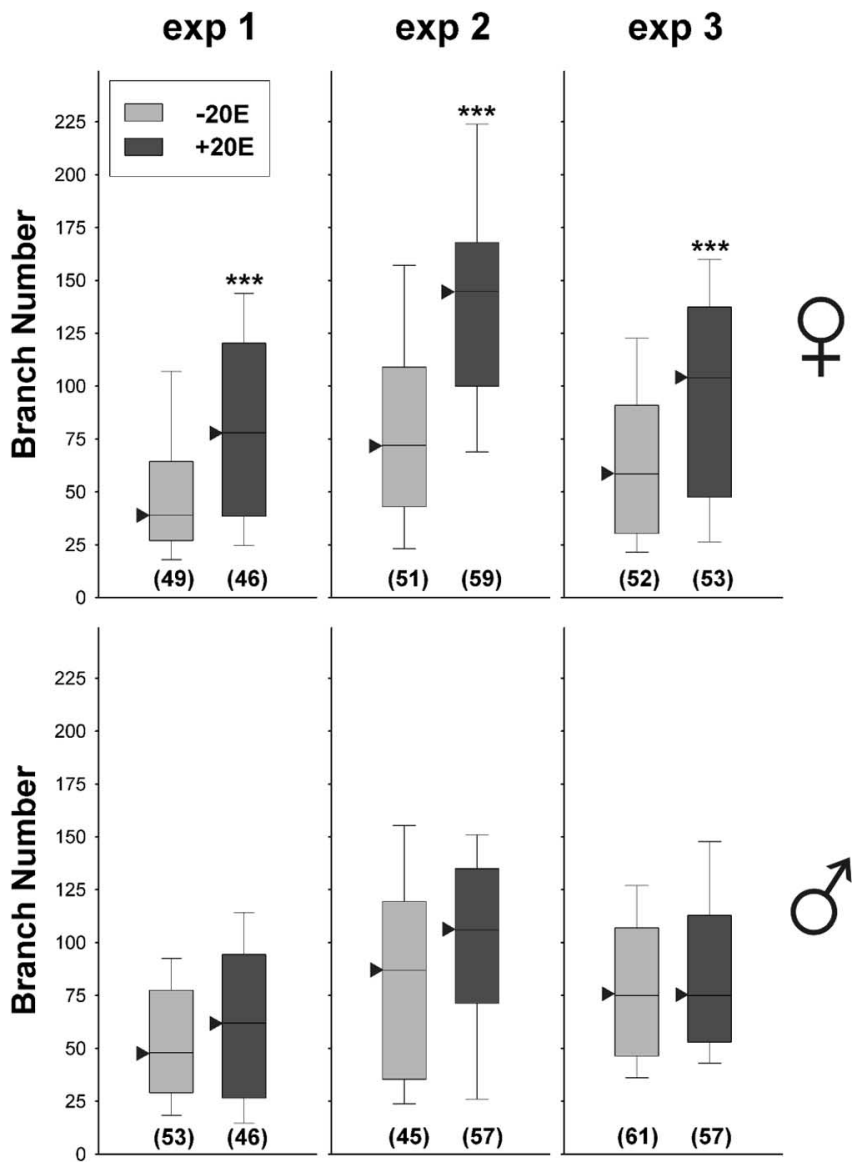

Figure 2. The response of pupal MB $\gamma$ neurons to $20 \mathrm{E}$ in vitro is sexually dimorphic. Box-plot distributions of branch-number data from three independent experiments comparing cultured $\gamma$ neurons from $201 Y$ UAS-lacZ/+ female-male sibling pairs. Neurons were dissociated at HE $+5 \mathrm{~h}$, and the preparation was split and cultured in the presence $(+)$ or absence $(-)$ of $20 \mathrm{E}$ for 3 div. The tops and bottoms of the boxes represent the 75 th and 25 th percentiles, respectively; triangles indicate the medians; and bars indicate the 10th and 90th percentiles. The number of neurons in each sample is shown in parentheses. All three female samples (top row) showed a highly significant effect of $20 \mathrm{E}$ on branch number $\left({ }^{* * *} p<0.0001\right.$ ), whereas $\gamma$ neurons from their brothers (bottom row) showed no significant response to the hormone ( $p=0.35,0.07$, 0.47 , respectively).

ends of microtubules. A fusion protein, Khc: $\beta$ gal, consisting of the plus-end-directed microtubule motor protein, Kinesin heavy chain, and $\beta$-galactosidase, is an in vivo plus-end reporter, labeling axons of embryonic sensory neurons but only poorly labeling their dendrites (Clark et al., 1997). In contrast, Nod: $\beta$ gal, containing the motor domain of Nod, a minus-end-directed microtubule motor, is an in vivo minus-end reporter, accumulating in cell bodies and dendrites but not in axons (Clark et al., 1997). In

$\leftarrow$

primary process. $\boldsymbol{B}$, Negative image of the neuron in $\boldsymbol{A}$, overlaid with a skeleton of pixels (green) and bounded by the perimeter of its territorial footprint (purple). The original one-pixel-wide skeleton was thickened to three pixels wide for greater visibility in this image. C, Box-plot distributions of total neurite length of $201 \gamma$-marked $\gamma$ neurons from female pupal brains isolated at $\mathrm{HE}+5 \mathrm{~h}$ and cultured 3 div in the presence $(+20 \mathrm{E}$; light gray) or absence $(-20 \mathrm{E}$; dark gray) of 20E. Black triangles indicate the medians; the tops and bottoms of the boxes represent the 75th and 25th percentiles, respectively; and the crossbars indicate the 90th and 10th percentiles. The number of neurons analyzed is indicated in parentheses below each plot. Left, Wild type (201Y/UAS-lacZ; $+/+$ ). The $p$ value of $<0.0001$, obtained using the Mann-Whitney rank-sum test indicates a highly significant response of total neurite length to $20 \mathrm{E}$ treatment. Right, E74A mutant [201Y/UAS-lacZ; E74A ${ }^{\text {Pneo }}$ mwh red $\left.e^{s} / D f(3 L) s t-81^{k 19}\right]$. The effect of $20 \mathrm{E}$ treatment on neurite length was not significant (n.s.) ( $p=0.07)$. 

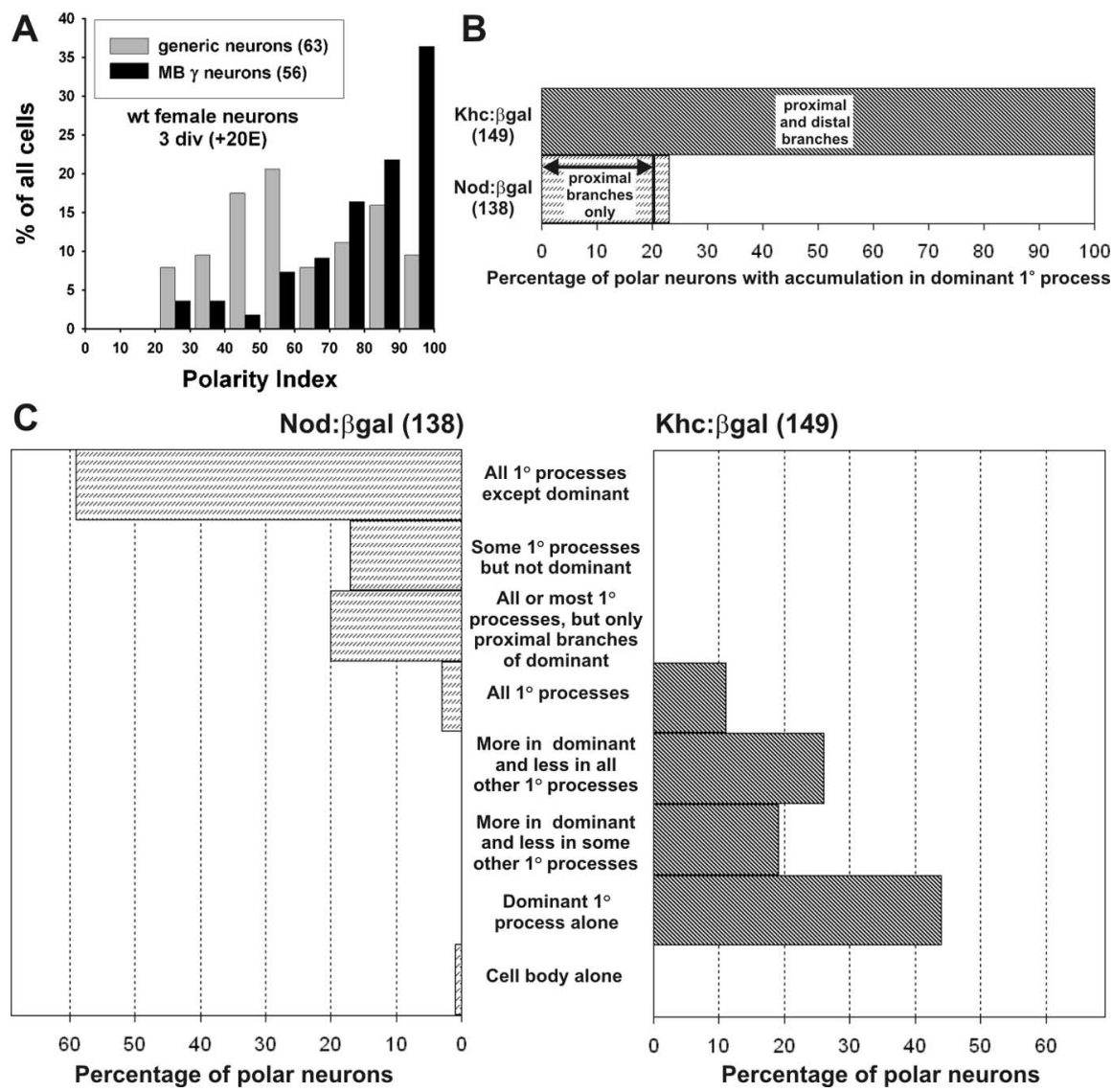

Figure 3. The polarity of $\mathrm{MB} \gamma$ neurons in vitro results from distinct axonal and dendritic arborizations. $\boldsymbol{A}$, Representative example of Polarity Index distributions of cultured $\gamma$ neurons and their generic neighbors isolated from the same wild-type pupal brain. Note the skewed distribution of PI values of MB $\gamma$ neurons, which reflect their highly polar neurite arbors. $B, C, C$ mparison of Nod: $\beta$ gal and Khc: $\beta$ gal fusion-protein distributions in subsets of neuronal processes when expressed in $\gamma$ neurons in vitro. Neurons were isolated from w; 201 Y UAS-GFP/+;UAS-Nod:lacZ/+ or w;201YUAS-GFP/+;UAS-Khc:lacZ/+ pupae at HE $+5 \mathrm{~h}$. Cells were cultured for $3 \mathrm{~d}$ and then fixed and double immunostained to detect all neuronal membranes and $\beta \mathrm{gal}$. $\boldsymbol{B}$, Comparison of Khc: $\beta$ gal and Nod: $\beta$ gal accumulation in the dominant primary processes of $\gamma$ neurons. Khc: $\beta g a l$ accumulates in all branches, whereas Nod: $\beta \mathrm{gal}$, which labels the dominant primary process in only $23 \%$ of neurons, accumulates primarily in its proximal branches. C, Classification of Nod: $\beta$ gal and Khc: $\beta$ gal fusion-protein localization patterns. Every polar neuron from the population sample was classified in one of eight mutually exclusive categories, listed in the center column. Left, UAS-Nod-lacZ driven by $201 Y$ in 138 polar neurons ( $83 \%$ of all labeled neurons in the sample). Right, UAS-Khc-lacZ driven by $201 Y$ in 149 polar neurons ( $86 \%$ of all labeled neurons in the sample).

addition, when expressed in adult MB neurons, Nod: $\beta$ gal accumulated in the calyx, a dense mass of dendritic arborizations, but not in the axons of the peduncle and lobes (Lee et al., 2000b).

We used $201 Y$ to drive expression of UAS-Nod-lacZ or UAS$K h c-l a c Z$ in cultured pupal MB $\gamma$ neurons and classified them based on the accumulation of each fusion protein within the neurite arbor (visualized by anti-HRP), with special attention to the dominant primary process. Of $271 \gamma$ neurons analyzed, $85 \%$ were "polar," meaning that they had an obvious dominant primary process. Within these polar $\gamma$ neurons, Nod: $\beta$ gal and Khc: $\beta$ gal had distinct and complementary distributions (Figs. 3, 4).

For both markers, fusion-protein distribution within neurites was usually nonuniform, with greater accumulation closer to branch termini (Fig. 4). As in vivo, Nod: $\beta$ gal accumulated in all neuronal cell bodies, with $98 \%$ of polar $\gamma$ neurons also showing accumulation in processes (Figs. $3 C$, left, $4 A, B$ ). When $\gamma$ neurons expressed Khc: $\beta$ gal, relatively little fusion protein was detected in cell bodies (Fig. 4C,D), but $100 \%$ of polar $\gamma$ neurons showed accumulation in their dominant primary processes (Fig. 3B). Specifically, Khc: $\beta$ gal was detected exclusively in the dominant primary process in $44 \%$ of neurons, with an additional $44 \%$ showing greater Khc: $\beta$ gal accumulation in the dominant primary process than in other primary processes (Fig. 3C, right). In contrast, in 75\% of polar $\gamma$ neurons, the Nod: $\beta$ gal distribution did not include the dominant primary process at all (Fig. 4C, left). The most common pattern (59\%) was Nod: $\beta$ gal accumulation in all primary processes except the dominant (Fig. 4A). In a minority of neurons (23\%), Nod: $\beta$ gal accumulated in the dominant primary process but did so in a highly selective manner, usually in only the most proximal branches (Figs. $3 B, C, 4 B)$.

In summary, cultured $\gamma$ neurons usually have a polar morphology, within which Khc: $\beta$ gal selectively labels the dominant primary process. Conversely, Nod: $\beta$ gal selectively labels the short primary processes and is excluded from the distal trunk and arbor of the dominant primary process. Based on the in vivo validation of these markers (Clark et al., 1997; Lee et al., 2000b), our in vitro data indicate that the dominant primary process represents the axon, whereas the short primary processes, as well as proximal branches of the dominant primary process, represent the dendritic arbor of the $\gamma$ neurons in vitro. Hence, the distinctive PI profile of cultured $\gamma$ neurons reflects an endogenous morphogenesis program, with a strategy of preferential investment in axonal growth. In addition, wild-type $\gamma$ neurons elaborate neurite arbors in vitro with a fixed lengthto-branch-number ratio that is unaffected by $20 \mathrm{E}$ exposure (Kraft et al., 1998) (data not shown). When wild-type morphogenesis in vitro is so constrained, mutant phenotypes are readily detected, as we demonstrate below.

\section{MB-restricted "naked runt" phenotype revealed in vitro}

We examined the transgenic line Dmef2-lac Z, with the MBspecific enhancer of Dmef2 fused to lacZ (Schulz et al., 1996), hoping it would be a useful in vitro marker. Anti- $\beta$ gal immunostaining of brains from larvae, pupae, and pharate adults revealed highly selective expression in the MBs and normal-appearing $\mathrm{MB}$ morphology (Fig. 5A-C). Double-labeling experiments with 201Y/UAS-GFP; Dmef2-lacZ in vivo showed almost complete overlap between the two reporter genes (data not shown). This, in combination with the apparent axon degeneration in Dmef2lacZ-labeled pupal MB lobes (Fig. 5B, arrows), indicates that the $D m e f 2$ enhancer is expressed primarily in $\gamma$ neurons. However, when young pupal brain neurons homozygous for Dmef2-lac $Z$ on the third chromosome were cultured, the morphology of the $\beta$ gal-expressing cells was strikingly abnormal (Fig. 5D,E) compared with wild-type neurons marked with $201 Y$-driven lacZ (Fig. 5F). Dmef2-lacZ-expressing neurons were reduced in size and had very few branches, inspiring the name "naked runt." 
The naked runt phenotype is restricted to Dmef2-lacZ neurons marked by $\beta$ gal expression. They were much smaller than the $201 Y$-marked neurons and had fewer branches (data not shown), whereas unmarked generic brain neurons of the two genotypes showed no differences in length (Fig. 5G). In addition, the marked neurons in Dmef2-lac Z cultures were much less polar than the $201 Y$-marked neurons (Fig. $5 \mathrm{H}$ ), with a PI profile similar to that of wild-type generic brain neurons (Fig. 3A), suggesting that isolated $\gamma$ neurons are no longer able to manifest a normal morphogenesis program. The naked runt phenotype was also seen with a single copy of the third-chromosome Dmef2-lacZ transgene (data not shown), indicating that it is dominant. However, a secondchromosome insertion of the same transgene did not produce the naked runt phenotype (data not shown). Thus, the third-chromosome Dmef2-lacZ transgene insertion dramatically disrupts in vitro $\mathrm{MB}$ neuron morphology, although in vivo $\mathrm{MB}$ morphology appears normal. Therefore, naked runt is a cryptic phenotype.

"Filagree" phenotype reveals a role for Fascin in neurite morphogenesis

While investigating the effect of X-linked mutations on pupal $\gamma$ neurons in vitro, we observed that cells from control siblings carrying the Binsn balancer exhibited a novel, distinctive morphology that we named "filagree" because of its resemblance to filagree jewelry and other ornamental metalwork (Fig. 6). In wild-type cultures, neurons show an overall clockwise growth pattern, most obvious along the path of the large axonal process (Figs. $1 A, 4,6 A$ ), with higher-order branches being relatively straight, even spike-like. In contrast, the neurites of Binsn-bearing $\gamma$ neurons curved clockwise in tight curls or hooks, often with variable and excessive width (Fig. $6 B, C$ ). Directional growth of neurites has been reported for other cultured neurons as well (Romijn et al., 1980) and may involve interaction between the substrate and the neuronal cytoskeleton (Farmer et al., 1992).

The filagree phenotype is cryptic, because the MBs that gave rise to it were normal by whole-mount immunostaining (detecting 201Y-driven $\beta$ gal expression) over a range of developmental stages, including their reorganization during metamorphosis (data not shown). The filagree phenotype was neither restricted to $\gamma$ neurons (Fig. 6E) nor developmental stage (Fig. $6 F, G$ ). At both larval and pupal stages, the extreme-curvature morphology
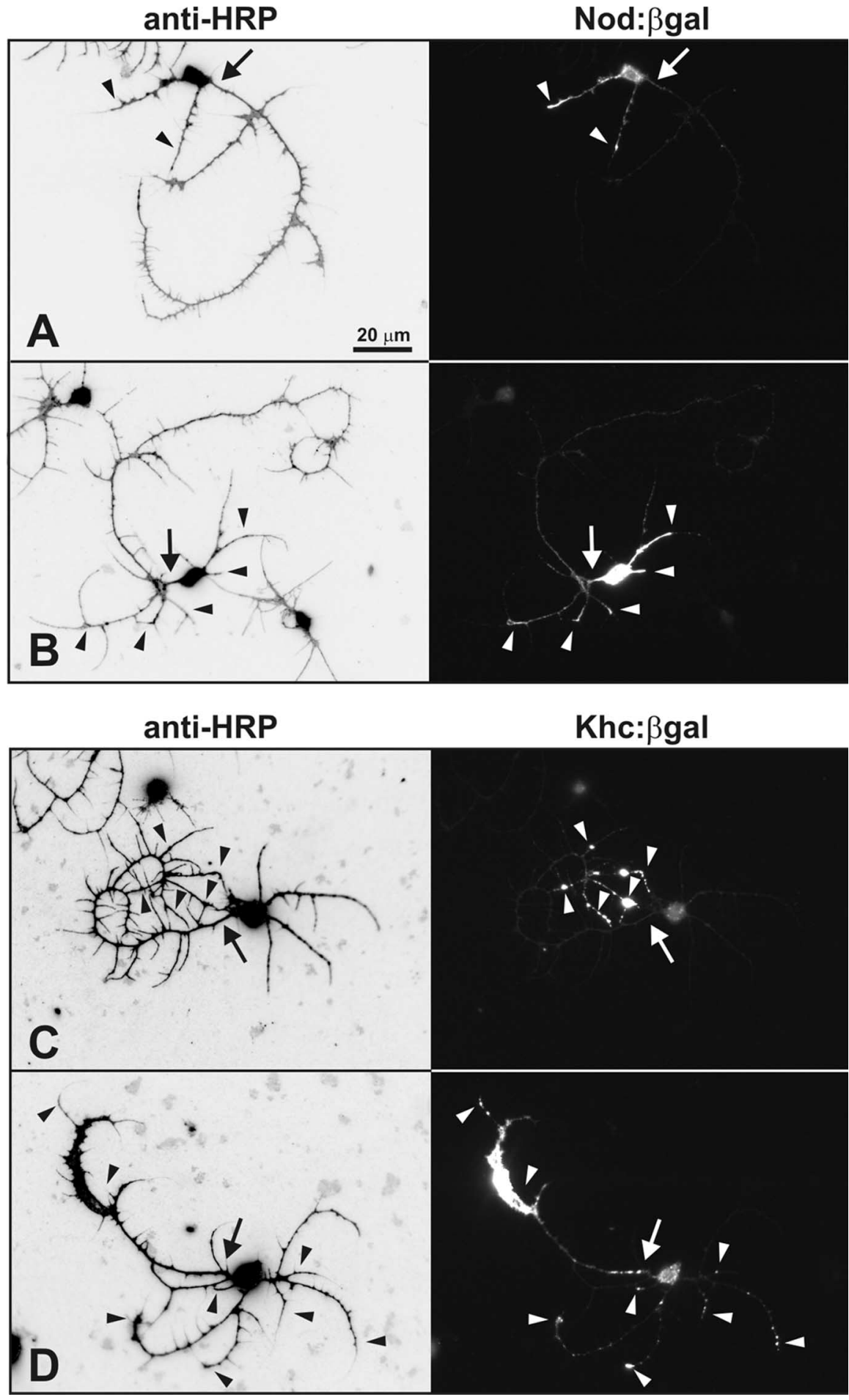

Figure 4. Nod: $\beta$ gal and Khc: $\beta$ gal distribution in $M B \gamma$ neurons in vitro. Examples of common distribution patterns of the fusion proteins within cultured $\gamma$ neurons with polar morphologies. Pupal neurons were prepared, cultured for 3 div, and stained as described in Figure 3. Two immunofluorescent images of each neuron are shown: left, negative anti-HRP image, showing neuronal membranes; right, positive anti- $\beta$ gal image, showing fusion protein accumulation. Arrows indicate the dominant primary process; triangles denote processes with Nod: $\beta$ gal or Khc: $\beta$ gal accumulation. The scale bar in $\boldsymbol{A}$ applies to all images. $\boldsymbol{A}$, $B, w ; 201 Y$ UAS-GFP/+; UAS-Nod:lacZ/+.$A$, Nod: $\beta$ gal in all primary processes except the dominant primary process, the most common pattern of Nod: $\beta$ gal distribution. $B$, Nod: $\beta$ gal in all or most primary processes. but in only the most proximal branches of the dominant primary process. C, D, w; 201YUAS-GFP/+;UAS-Khc:lacZ/+ .C, Khc: $\beta$ gal in the dominant primary process alone, the most common pattern of Khc: $\beta$ gal distribution. D, More Khc: $\beta \mathrm{gal}$ in the dominant primary process and less in all others. 

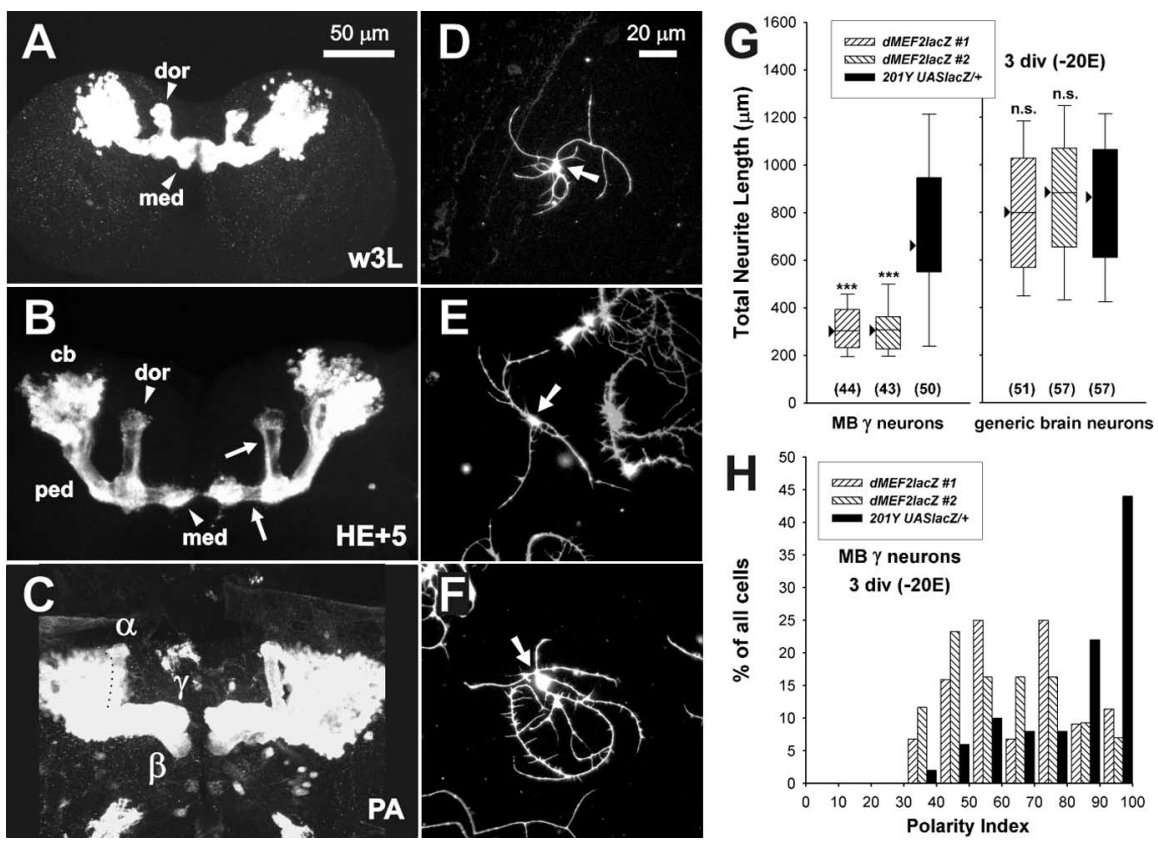

Figure 5. A naked runt phenotype of $\mathrm{MB} \gamma$ neurons is unmasked in vitro. A-C, Normal morphology of MBs in female Dmef2lacZ homozygotes (third chromosome insertion). Projections of sequential optical sections through the MBs of anti- $\beta$ gal immunostained brains. The scale bar in $\boldsymbol{A}$ applies to $\boldsymbol{A}-\boldsymbol{C} . \boldsymbol{A}$, w3 L, Wandering third instar larva, with normal-appearing medial (med) and dorsal (dor) MB lobes. $\boldsymbol{B}, \mathrm{HE}+5 \mathrm{~h}$, Young pupa. Reduced signal intensity in the lobes (arrows) indicates stage-appropriate degeneration of $\gamma$ neuron axons. cb, Cell body cluster. C, PA, Pharate adult, several hours before adult emergence, with normalappearing $\alpha, \beta$, and $\gamma$ lobes. The left $\alpha$ lobe is partially obscured by the cell body cluster. $\boldsymbol{D}-\boldsymbol{H}$, Cultured female pupal MB neurons, isolated at HE $+5 \mathrm{~h}$, cultured for 3 div with media lacking $20 \mathrm{E}$, and visualized by anti-HRP immunofluorescent staining. Arrow, Cell body. The scale bar in $\boldsymbol{D}$ applies to $\boldsymbol{D}-\boldsymbol{F}$. D, E, Representative $\beta$ gal-positive (MB $\gamma$ ) neurons homozygous for Dmef2lacZ (third chromosome). Note the small size and paucity of branches of the neurite arbors. $\boldsymbol{F}$, Representative $\gamma$ neuron from a parallel wild-type (201Y UAS-lacZ/+) culture. Note the considerable length and large number of branches. G, Box plots comparing total neurite lengths of Dmef2-lacZ homozygotes (two independent experiments, 1 and 2) with those of 201Y UAS-lacZ/+ . The left side shows $\gamma$ neurons, identified by $\beta$ gal expression; the right side shows generic brain-neuron neighbors from the same cultures. The number of neurons in each sample is shown in parentheses. $\beta$ gal-expressing Dmef2-lacZ neurons are greatly reduced in total length compared with control $\gamma$ neurons $(p<0.0001)$, whereas there is no significant (n.s.) difference in the length distributions between generic neurons of the two genotypes. $\boldsymbol{H}$, Polarity Index distributions for the $\gamma$ neurons shown in $\mathbf{G}$. The control (201Y UAS-lacZ/+) population exhibits the typical wild-type skewed distribution, whereas $\beta$ gal-expressing Dmef2lacZ neurons have very reduced polarity.

was fully penetrant, detectable in most processes of all brain neurons in the cultures. The penetrance and expressivity of the excess-width feature were incomplete and highly variable, respectively, among filagree neurons.

The 20E responsiveness of female pupal $\gamma$ neurons exhibiting the filagree phenotype was not compromised. Total neurite length (Fig. $6 \mathrm{H}$, left) as well as branch number and territory (data not shown) were enhanced by $20 \mathrm{E}$ to an extent comparable with the wild-type outgrowth response to the steroid hormone. However, for a given hormone-treatment condition, filagree $\gamma$ neurons covered significantly less territory than did wild-type neurons (Fig. $6 \mathrm{H}$, middle) and, conversely, branch-point density was significantly higher (Fig. $6 \mathrm{H}$, right). In other words, for cells of a given total length, filagree neurons had smaller territories and greater branching per unit area because of their extraordinary curvature (Fig. 6I). Despite their filagree phenotype, the $\gamma$ neurons displayed a normal PI profile (Fig. $6 J$ ), with most of the cells having a large dominant primary process. Based on our analysis of axon and dendrite markers (Figs. 3, 4), the normal PI profile suggests that the filagree phenotype does not disrupt the balance of axon-dendrite morphogenesis.

A retrospective review of many cell culture experiments revealed that the filagree phenotype appeared whenever neurons were homozygous mutant for singed (sn). We obtained stocks of two independent alleles, $s n^{X 2}$ (the $\mathrm{x}$-ray-induced allele on the Binsn balancer) and the spontaneous mutation $s n^{3}$, and tested several genotypes. Cultured brain neurons of the genotypes $s n^{3} / s n^{3}, s n^{3} / s n^{X 2}$, and $s n^{X 2} / Y$ from young pupae (Fig. $7 A-C$ ) or wandering third instar larvae (Fig. 7D-F) exhibited an obvious filagree phenotype. All brain neurons were affected, with more variation in the broadening of neurites than in the curvature. A commonly used X-linked recessive visible marker, singed (Fig. 7G) (Bender, 1960) (FlyBase FBgn0003447; http://flybase. org/) has been used to study the molecular and ultrastructural mechanism of bristle morphogenesis (Tilney et al., 1995). The filagree phenotype was easily detectable in living neurons after $3 \mathrm{div}$ (Fig. 7 H,I) and was already evident after just $24 \mathrm{~h}$ of culture (Fig. $7 J, K$ ). By visual inspection, the phenotypic severity was greatest in $s n^{X 2} / Y$ and least in $s n^{3} / s n^{3}$, with $s n^{3} / s n^{X 2}$ being intermediate, regardless of other markers or mutations. This is consistent with classification of $s n^{X 2}$ as more severe (probably null) than $s n^{3}$ (hypomorphic) based on bristle phenotype (Bender, 1960) and molecular criteria (Paterson and O'Hare, 1991; Cant et al., 1994). Hence, the filagree phenotype of cultured brain neurons maps to the singed locus.

\section{Fascin mutations cause abnormal actin distribution in neurons}

The singed gene encodes a member of the Fascin family of actin-bundling proteins (Bryan et al., 1993). Mammalian fascins 1, 2 , and 3 are encoded by three paralogous genes, of which FSCN1 is most similar to Drosophila singed (Kureishy et al., 2002). During bristle morphogenesis, fascin cross-links filamentous actin (F-actin) into bundles that prefigure the mature bristle shape (Cant et al., 1994; Tilney et al., 1995). When fascin is missing or greatly reduced, the bundles are poorly organized and irregularly spaced, resulting in the gnarled and twisted singed bristle phenotype (Guild et al., 2003; Tilney and DeRosier, 2005). If the curling of filagree neurites is the counterpart of the twisting of $s n$ mutant bristles, then F-actin should be abnormally distributed within $s n$ mutant neurites.

To visualize the organization of F-actin within cultured neurons, we double-labeled them with anti-HRP and BODIPY FL phallicidin, which selectively and stoichiometrically binds to F-actin (De La Cruz and Pollard, 1994). In wild-type neurites, F-actin is distributed as nonuniform slender strands, with foci of increased density and thickness in cell bodies and presumed growth cones (Fig. $8 A, B$ ). In contrast, in $s n^{3} / s n^{X 2}$ mutant neurons, $\mathrm{F}$-actin is much more abundant in the neurites and presumed growth cones (Fig. 8C-G), with dense, cord-like accumulations adjacent to the plasma membrane. Thus, greatly reduced levels of fascin result in the abnormal distribution of F-actin within singed mutant neurons. As is the case for bristle morphogenesis (Tilney and DeRosier, 2005), this disruption of the actin 
cytoskeleton may underlie the excessively curved and variably widened neurites that characterize the filagree phenotype.

\section{Neurite curvature quantitation distinguishes singed and wild-type neuronal populations} The striking difference between filagree and wild-type phenotypes suggested they might be distinguishable, on a cell-by-cell basis, by observers who were blind to the neuron genotypes. Images of cultured pupal neurons from $s n^{3} / s n^{X 2}$ and $+/+$ (OreRC) brains were thoroughly shuffled and sorted by laboratory members into "normal" and "filagree" categories. Seventy-three percent of the $s n^{3} / s n^{X 2}$ neurons $(n=85)$ were classified as filagree, $18 \%$ as normal, and $9 \%$ as indeterminate. Of the OreRC cells $(n=48), 94 \%$ were classified as normal, $2 \%$ as filagree, and $4 \%$ as indeterminate. Thus, neurons classified as filagree were almost always $(98.4 \%$ accuracy) mutant in genotype. Mutant neurons at the lower end of the severity spectrum were incorrectly classified or deemed indeterminate because their curvature fell within the wild-type range.

The relative success of this subjective classification test suggested that objective means of quantifying curvature would verify these population differences and, moreover, might serve as a basis for accurate phenotypic classification. We therefore developed an automated computerbased method using skeletonized images to quantify overall neurite curvature. Curvature was calculated for each point along the neuron skeleton (Fig. 9A-D), and the values were averaged over the whole skeleton to generate "mean curvature," $|\bar{\kappa}|$, for each neuron. We applied this algorithm to skeletons of $s n^{+}$and $s n$-mutant neurons cultured with or without 20E. Figure 9, E and $F$, shows the frequency distributions of mean curvature values for the four data sets (the neurons came from the experiment depicted in Fig. 6). Under both culture conditions, $s n$-mutant neurons have much higher curvature values than do wild-type neurons. Under the assumption of normality, the differences between the two means are significant in both cases at $p=0.0005$ using Welch's $t$ test to account for different variances and sample sizes.

The very significant differences of the curvature distributions suggested that we might be able to classify neurons automatically into either mutant or normal categories. Table 1 shows classification results obtained using leave-one-out cross-validation and a calculated decision boundary (see supplemental Materials and Methods, available at www.jneurosci.org as supplemental material) and based on the assumption of normality of the population distributions. The overall classification accuracy for the two ex-
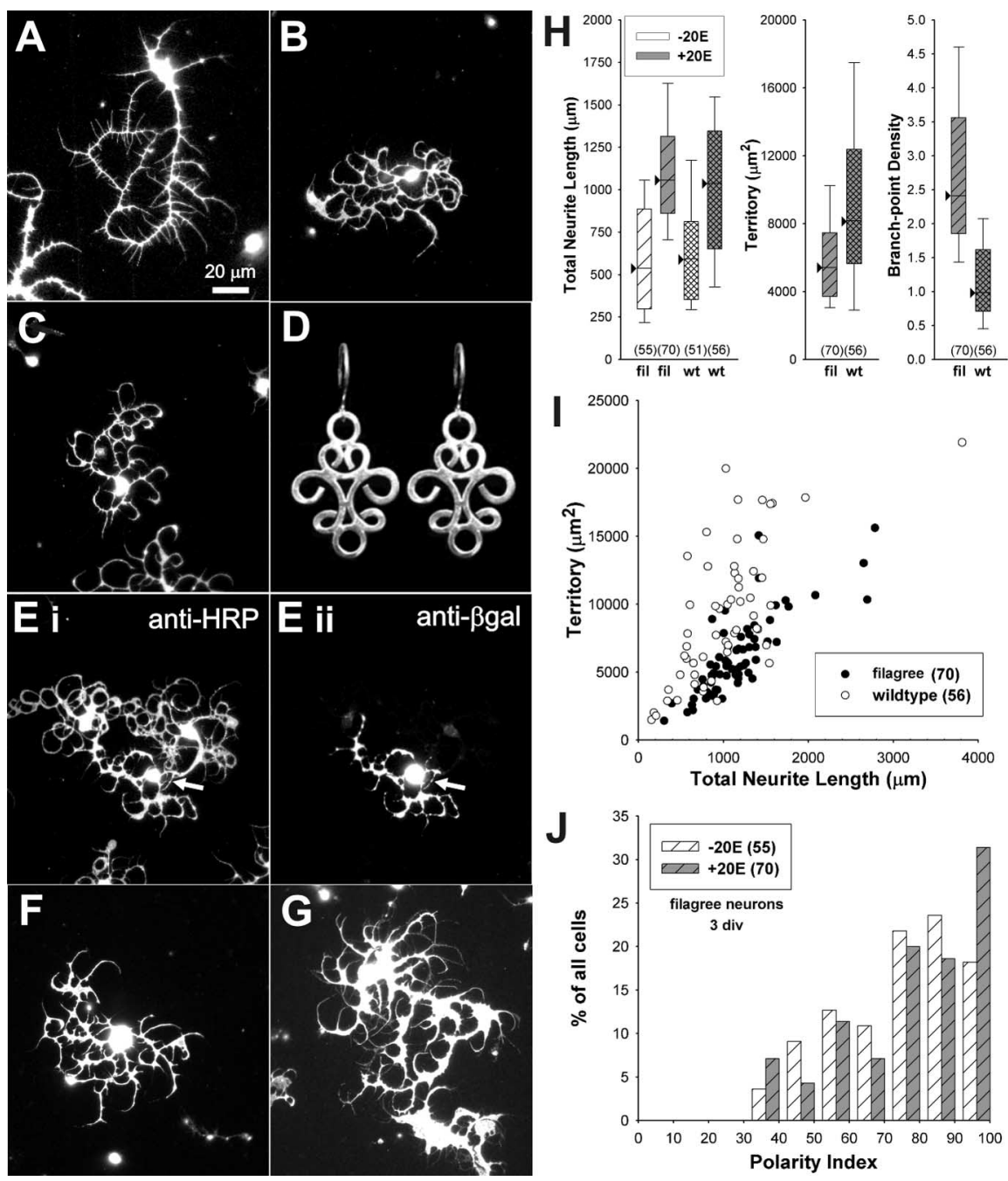

Figure 6. Filagree phenotype identified in brain neurons in primary cell culture. Female brain neurons, harvested from wandering third instar larvae or young pupae (HE $+5 \mathrm{~h}$ ), cultured 3 div, and visualized by anti-HRP immunostaining. $\gamma$ neurons were identified by $201 Y$-driven $\beta$ gal expression. $\boldsymbol{A}$, Wild-type pupal $\gamma$ neuron (201Y UAS-lacZ/+). Note the overall clockwise growth pattern of the largest branches but the spike-like appearance of the terminal neurites. $\boldsymbol{B}, \boldsymbol{C}$, Representative pupal $\gamma$ neurons manifesting the filagree phenotype ( $y n p r 1^{3} w s n^{3} /$ Binsn; 201 Y UAS-lacZ/+). The phenotype includes striking curvature of nearly all neurites, irregular neurite caliber, and the hook-like appearance of many branch termini. $\boldsymbol{D}$, Example of filagree jewelry. $\boldsymbol{E}$, Pupal brain neurons ( $y$ npr13 w sn $^{3}$ /Binsn; $201 Y$ UAS-lacZ/+) need not be MB $\gamma$ neurons to show the filagree phenotype. Anti-HRP (Ei) and anti- $\beta$ gal (Eii) images of the same field. A single cell is $\beta$ gal-positive (arrow), but all neurons in the field display the filagree phenotype. $\boldsymbol{F}, \mathbf{G}$, Anonymous larval brain neurons ( $y n p r 1^{3} \mathrm{~W} \mathrm{sn}^{3}$ /Binsn) also manifest the filagree phenotype. $\boldsymbol{H}-\boldsymbol{J}$, Morphometric comparison of female pupal $\gamma$ neurons with and without the filagree phenotype ( $y r_{b p}^{5} w^{a} s n^{3} / B i n s n ; 201 Y$ UAS-lacZ/+ and 201Y UAS-lacZ/+, respectively). The number of neurons in each sample is shown in parentheses. $\boldsymbol{H}$, Box plot distributions of total neurite length (left), territory (center), and branch-point density (right). Both wild-type (wt) control neurons and filagree (fil) neurons respond to 20 E with increased total neurite length ( $p<0.0001$ for each). For a given culture condition, there was no significant difference between the length distributions of wild-type and filagree neurons. However, filagree neurons cover smaller territories ( $p<0.0001)$ and have higher branch-point densities $(p<0.0001)$ than do wild-type neurons. $I$, Length-territory relationships. For a given total length, filagree neurons cover less territory than do wild-type neurons. J, Polarity Index distribution of the filagree neurons. In both the presence and absence of $20 \mathrm{E}$, the profiles were very similar to wild-type (Fig. $3 A)$, with the majority of neurons having a highly polar morphology.

periments was $92 \%$. Future use of additional morphometric features, such as neurite width, might further increase classification accuracy.

\section{Discussion}

Fidelity of MB $\gamma$-neuron morphogenesis and hormoneregulated plasticity in vitro

A primary neuronal culture system using Drosophila MB $\gamma$ neurons harvested from young pupal brains recapitulates features of normal morphogenesis and developmental plasticity and allows 

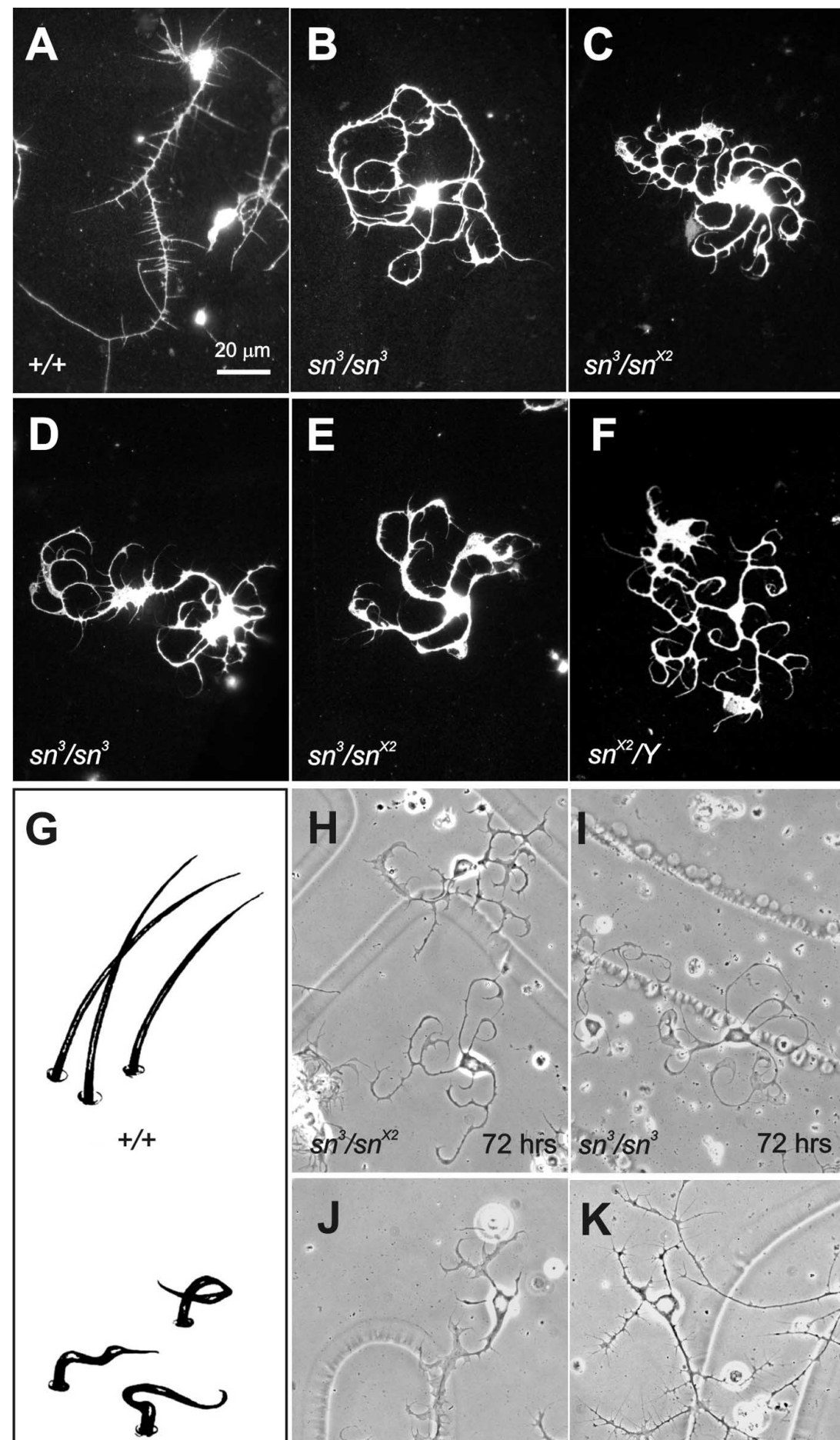

$s n$

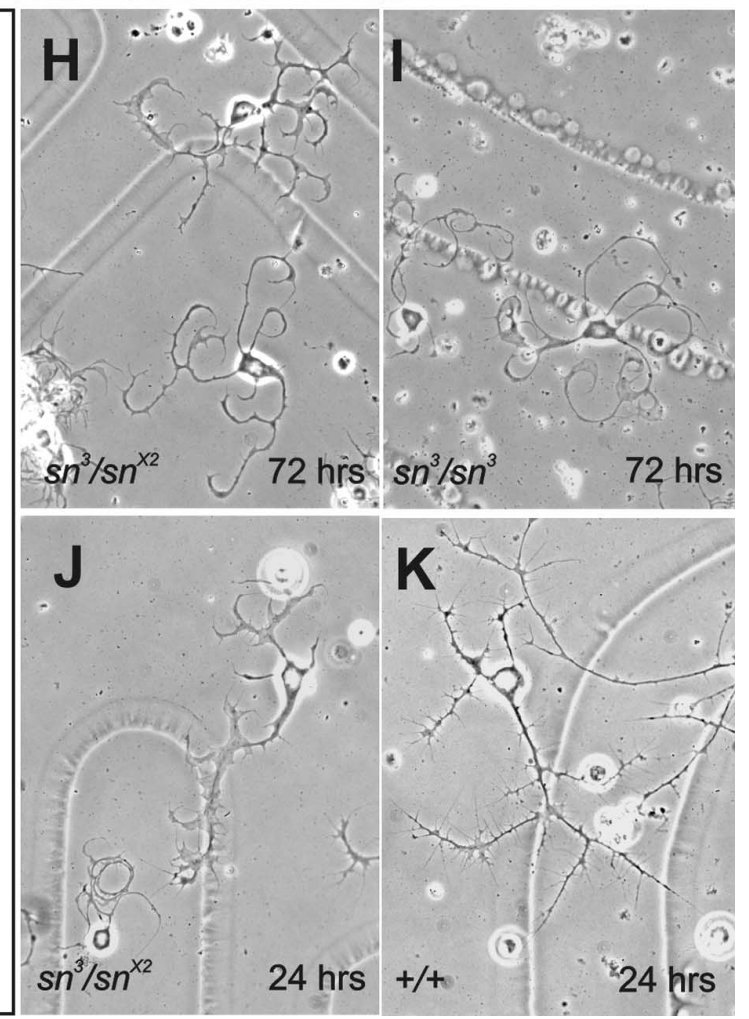

Figure 7. Loss of fascin, the actin-bundling protein encoded by singed (sn), causes the filagree phenotype. The scale bar in $\boldsymbol{A}$ refers to $\boldsymbol{A}-\boldsymbol{F}$ and $\boldsymbol{H}-\boldsymbol{K} . \boldsymbol{A}-\boldsymbol{F}$, Anonymous female brain neurons of the indicated genotypes and stages, cultured 3 div and visualized by anti-HRP immunostaining. $A-C$, Pupal neurons dissociated at HE $+5 \mathrm{~h} . A, 0 \mathrm{reRC}\left(\mathrm{sn}^{+} / \mathrm{sn}^{+}\right)$. Note the typical mild clockwise curvature of the dominant primary process, but the straight trajectory of most higher-order branches. $\boldsymbol{B}, \boldsymbol{C}$, singed mutant neurons display the filagree phenotype. $B, s n^{3} / s n^{3} . C, s n^{3} / s n^{X 2}$. D $-F$, Neurons from wandering third instar larvae. Note the severity range of the filagree phenotype: $s n^{3} / s n^{3}<s n^{3} / s n^{X 2}<s n^{X 2} / Y$. G, Drawings of bristle phenotypes, wild-type (top), and singed mutant (bottom), adapted from Carolina Biological Supply Company (1988) and used by permission. $\boldsymbol{H}-\boldsymbol{K}$, Live neurons from wandering third instar larvae, cultured for the indicated times, imaged with phase-contrast optics. $\boldsymbol{H}-\boldsymbol{J}$, singed mutant neurons show the filagree phenotype after 1 or 3 div. $\boldsymbol{K}$, Wild-type neurons (201Y UAS-lacZ/+). At $24 \mathrm{~h}$, the overall clockwise curvature is detectable, but most neurites, especially terminal branches, are straight. normal and mutant phenotypes to be revealed and quantified. Gamma neurons elaborate a neurite arbor with marked polarity and distinct axonal and dendritic components. The dominant primary process (strongly labeled by Khc: $\beta$ gal) is axonal. Most often, dendritic neurites (labeled by Nod: $\beta$ gal) emerge from the soma, as is typical of vertebrate neurons, but a considerable minority grow from the most-proximal shaft of the dominant primary process, analogous to the in vivo location of most insect dendrites (Strausfeld and Meinertzhagen, 1998). Such variation in dendrite position, as well as axonal identity of the longest primary process, has also been reported for Drosophila motor neurons in vitro (Sanchez-Soriano et al., 2005). Because axons and dendrites grow and remodel by distinctive mechanisms and are differentially affected by mutations (Lee et al., 2000b; Arimura and Kaibuchi, 2005), there is great potential value in being able to monitor them separately in vitro. The polar morphology of cultured $\gamma$ neurons makes it possible to use PI profiles as a rough indicator of axon-dendrite identity or differentiation. For instance, the abnormal PI profile of $d M e f 2-l a c Z$ neurons suggests that their $\gamma$ neurite-outgrowth defects do not affect axons and dendrites equally.

MB developmental plasticity includes two phases, both during peaks of 20E: degeneration of $\gamma$-neuron axons and dendrites at the end of larval life, followed by outgrowth of new processes during pupal life (Lee et al., 1999). This dramatic neuronal remodeling likely allows the MBs to serve adult-specific behavioral functions (e.g., memory and courtship) (Heisenberg, 2003). The degeneration phase is dependent on the dimeric 20E receptor (Lee et al., 2000a). Our previous in vitro study, demonstrating proportional enhanced outgrowth of $\gamma$ neurites in response to $20 \mathrm{E}$ treatment, supports the hypothesis that regrowth, which occurs while $20 \mathrm{E}$ levels are rising in vivo, is also hormone dependent (Kraft et al., 1998). Here, we have shown that E74A is an essential mediator of the outgrowth response to $20 \mathrm{E}$ in vitro. Consistent with our findings, E74A transcripts appear to be preferentially expressed in the MB cell-body regions of the early prepupal CNS [Boyd et al. (1991), their Fig. $1 F$ ]. Primary culture has allowed us to study healthy neurons from whole-animal mutants several days after harvesting, although the animal would have died shortly after harvesting. Our results conflict with an interpretation from genetic mosaic studies that $E 74 A$ is dispensable for $\mathrm{MB}$ 
remodeling (Lee et al., 2000a). The discrepancy may indicate that some E74A functions are not cell-autonomous.

\section{Unsuspected neuronal phenotypes revealed in vitro}

We found that 20E-regulated $\gamma$-neuron outgrowth is markedly sexually dimorphic despite the absence of gross differences between male and female MBs. This is reminiscent of sexually dimorphic estrogen effects on dendritic spines in behaviorally relevant mammalian brain regions (Miranda et al., 1999; Lee et al., 2004). Adult MB axon number is slightly smaller in males than in females (Technau, 1984), which suggests that sexually dimorphic hormone responsiveness during metamorphosis could underlie sexually dimorphic adult morphology. The mechanism responsible for the $\gamma$-neuron sex difference is likely to involve doublesex, a transcriptional regulator of sexual differentiation (Arbeitman et al., 2004; Christiansen et al., 2002), the expression of which in the pupal CNS is highly dynamic (Lee et al., 2002). Functional implications of these sex differences are suggested by evidence of $\mathrm{MB}$ involvement in sex-specific courtship behavior (O'Dell et al., 1995; Ferveur et al., 1997; Manoli et al., 2005) (but see Kido and Ito, 2002).

We found two other striking examples of phenotypes detected in vitro that were unexpected because of apparently normal MB morphology. The dominant, $\gamma$-neuronspecific naked runt phenotype may represent a gain-of-function mutation, resulting from ectopic activation of a gene in the vicinity of the third-chromosome Dmef2-lacZ insertion site. The recessive, global filagree phenotype is attributable to severe fascin deficiency caused by strong loss-of-function singed mutations. Mutant neurites show abnormal F-actin distribution, similar to that found in developing singed-mutant bristles (Guild et al., 2003). Microtubules may also be involved in the filagree phenotype, given that neurite trajectory (Kalil and Dent, 2005) and bristle morphogenesis (Tilney and DeRosier, 2005) involve interaction between actin and microtubule components of the cytoskeleton.

\section{Filagree: meaningful neuronal phenotype or} cell-culture artifact?

Despite the high penetrance of the filagree phenotype, overall brain organization in fascin-deficient singed mutants, assessed using well-characterized antibody markers and confocal microscopy, showed no obvious defects at several developmental stages (data not shown). We used markers to reveal subsets of axonal tracts or all synaptic neuropil or photoreceptor innervation of the optic lobes. It remains possible that we missed subtle defects or, perhaps even more likely, that higher-resolution methods of neuronal membranes
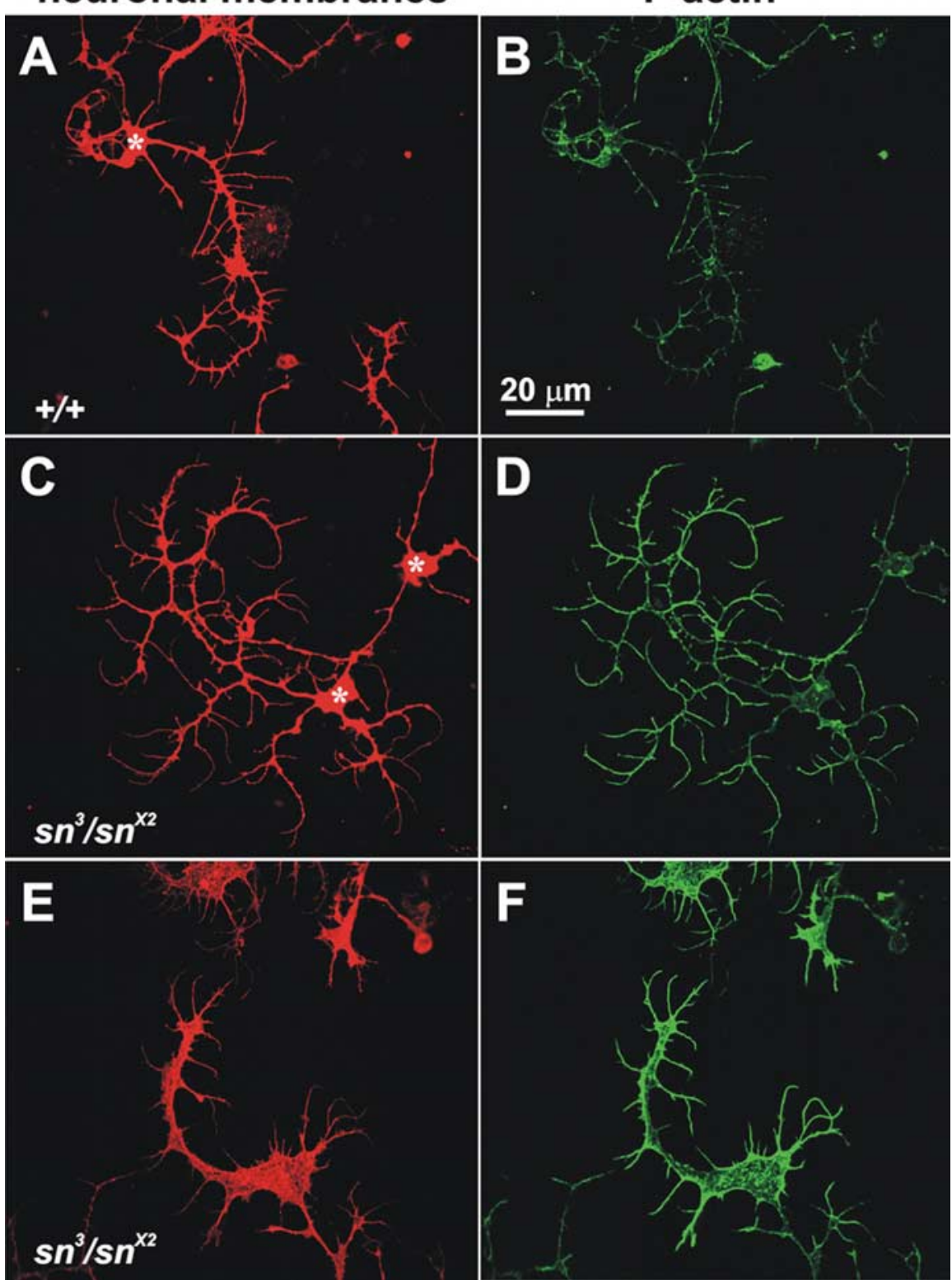

Figure 8. Filamentous actin distribution is altered in fascin-deficient filagree neurons. $\boldsymbol{A}-\boldsymbol{F}$, Neurons from female brains of wandering third instar larvae, cultured for 3 div, and double labeled with anti-HRP (left) to detect neuronal membranes and BODIPY FL phallicidin (right) to detect F-actin. Cell bodies are indicated by asterisks; the cell body of the neurons in $\boldsymbol{E}$ and $\boldsymbol{F}$ are outside the microscopic field shown. The scale bar in $\boldsymbol{A}$ applies to all images. $\boldsymbol{A}, \boldsymbol{B}$, Wild-type (201Y UAS-lacZ/+). F-actin is diffusely distributed throughout the processes with variable density that is slightly increased in the cell body and some branch termini. $\boldsymbol{C}-\boldsymbol{F}, s n^{3} / s n^{X 2}$. F-actin density is higher and more uniform than in wild-type neurites. In abnormally broad neurites $(\boldsymbol{E}, \boldsymbol{F})$, F-actin accumulation is especially prominent underlying the cell membrane. single-neuron labeling or electron microscopy will be required to detect singed-mutant neuronal phenotypes in vivo. Another possibility is that fascin regulation of the actin cytoskeleton in vivo controls the stability-lability balance at plastic synapses. F-actin localization at mushroom body postsynaptic sites (Frambach et al., 2004) and its dendritic-spine-specific modulation by activity at hippocampal synapses (Ouyang et al., 2005) are consistent with a role for fascin in synaptic plasticity. If true, then one might need high-resolution, real-time brain imaging to reveal dynamic defects in singed mutations.

The in vivo-in vitro difference (seemingly normal brain vs highly abnormal cultured neurites) may result in part from phe- 

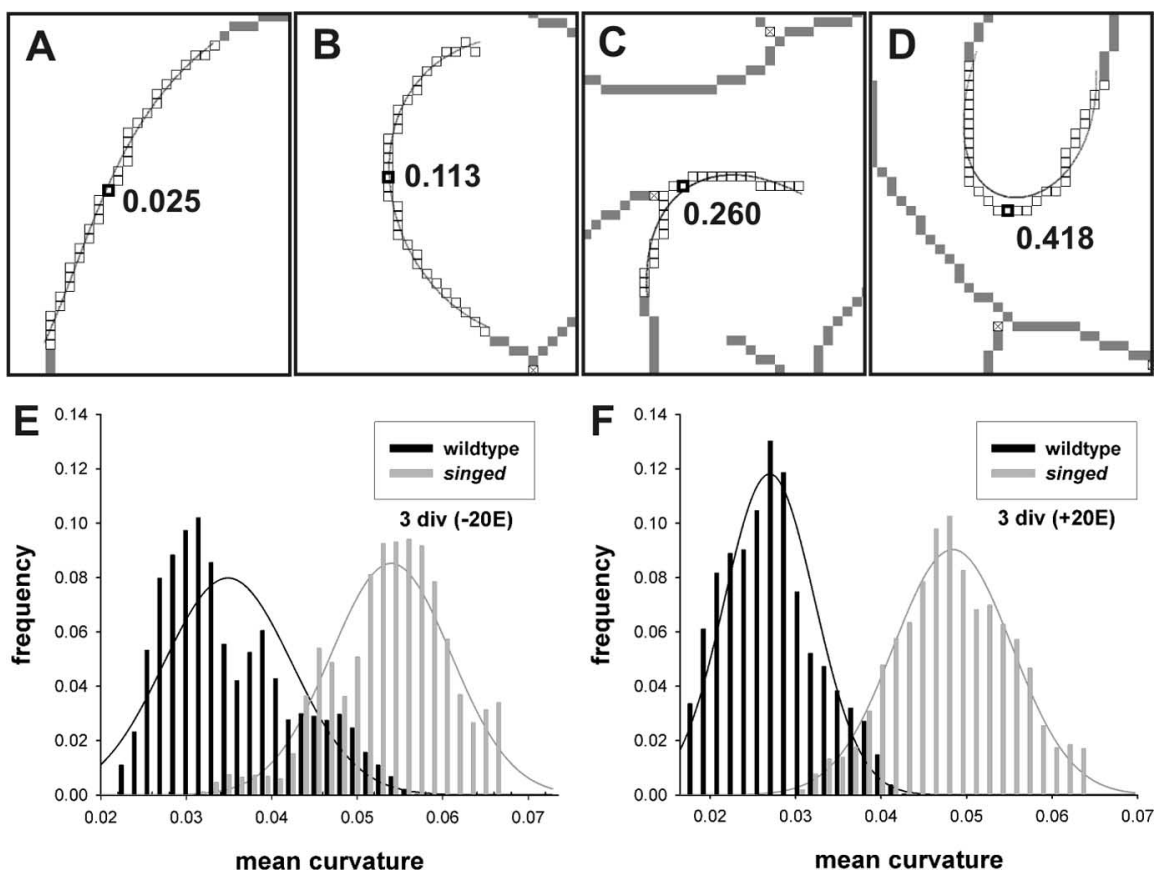

Figure 9. Curvature quantification distinguishes wild-type from fascin-deficient sn mutant neurons. $A-D$, Examples of curvature values of skeletonized neurite segments. The gray line represents the curve fitted to a segment that is centered on the dark pixel and flanked by the open pixels. Each value shown was computed for the indicated central pixel. Gray pixels were not included in that segment but were used to compute curvature of other segments; pixels marked $X$ were removed during skeleton processing. $\boldsymbol{E}, \boldsymbol{F}$, Frequency distributions of mean curvature values for pupal $\mathrm{MB} \gamma$ neurons cultured for 3 div in the absence $(\boldsymbol{E})$ or presence $(\boldsymbol{F})$ of $20 \mathrm{E}$. The histograms were plotted with soft binning, and normal distribution curves were fit to each population. Wild type, 201 Y UAS-lacZ/+ . singed, $y r_{b p}^{5} w^{a} s^{3} /$ Binsn; 201 Y UAS-lacZ/+.

Table 1. Automated classification of neurons based on computed mean curvature

\begin{tabular}{llllll}
\hline & \multicolumn{1}{c}{$(-) 20 \mathrm{E}$} & & & $(+) 20 \mathrm{E}$ \\
\cline { 2 - 3 } \cline { 5 - 6 } Phenotype classification & $\begin{array}{l}\text { wildtype } \\
(n=51)\end{array}$ & $\begin{array}{c}\text { singed } \\
(n=55)\end{array}$ & & $\begin{array}{l}\text { wildtype } \\
(n=54)\end{array}$ & $\begin{array}{l}\text { singed } \\
(n=70)\end{array}$ \\
\hline Normal & 44 & 5 & & 51 & 3 \\
Mutant (filagree) & 7 & $\mathbf{5 0}$ & & 3 & $\mathbf{6 7}$ \\
\hline
\end{tabular}

The columns show how many neurons of each genotype were predicted, based on curvature values, to belong to "normal" or "mutant" (filagree) phenotype groups. Each neuron image was held out from the training process, and a decision boundary was computed based on the two distributions. Then, the held-out neuron was classified according to that boundary. Accurate classification values are shown in bold.

notype amplification when dissociated neurons are cultured in relative isolation. For example, in the culture dish, mechanical tension between the neuron and its substrate (protein-coated glass) may be much greater than that generated between neurons and the extracellular matrix surrounding them in vivo. This difference also raises the question of whether the cell-culture phenotype is relevant to in vivo function. We believe that the available evidence and historical precedent argue persuasively that it is.

First, the filagree phenotype parallels the singed bristle phenotype, both in its morphological features and the accompanying disruption of the F-actin cytoskeleton, reflecting an essential role for fascin in actin bundling in neurons and bristle cells. The importance of the actin cytoskeleton is well-established for neuritogenesis, growth cone dynamics, and synaptic plasticity (Luo, 2002; Dillon and Goda, 2005). Second, expression data are strongly suggestive of a role for fascin in neuronal morphogenesis. The localization of fascin to growth cone filopodia (Sasaki et al., 1996; Cohan et al., 2001) suggested to the latter authors that fascin promotes "the linear structure and dynamics of filopodia." The failure of singed-mutant neurites to maintain a straight tra- jectory and uniform caliber provides compelling experimental support for this hypothesis. In mammals, FSCN1 is highly expressed throughout the entire developing CNS (De Arcangelis et al., 2004) and is upregulated in differentiating neurons (Megiorni et al., 2005). Third, although FSCN1 mutations have not yet been reported, FSCN2, which is retinal photoreceptor-specific, is required for maintenance of neuronal integrity. Lossof-function mutations cause dominant retinitis pigmentosa or macular degeneration (Wada et al., 2001, 2003), indicating that photoreceptors cannot tolerate a 50\% reduction in gene dosage.

Finally, we note the well-known example of a "cell-culture artifact" revealing a functionally relevant but otherwise undetectable phenotype: fragile sites, the appearance of which in the metaphase chromosomes of cultured lymphocytes depends on use of folate-deficient culture medium (Sutherland, 1983). In particular, an X-chromosome fragile site became a diagnostic marker for the most common form of inherited mental retardation and led to identification of mutations in FMR1 (Bardoni et al., 2000), a gene essential for brain development and function in fruit flies as well as humans (Michel et al., 2004; Zarnescu et al., 2005). Specifically, altered chromatin structure at the fragile-X site in vitro reflects the molecular pathogenesis but cannot be detected by microscopic examination of cells in vivo.

FSCN1 is a candidate gene for developmental brain disorders In nonneuronal cells, fascin-1 plays numerous roles in the formation of protrusions that regulate adhesion and motility, including tissue invasion by tumor cells (Peraud et al., 2003; Adams, 2004; Roma and Prayson, 2005). Together, the available data suggest that fascin also plays an essential role in CNS development and function. We predict that singed-mutant flies will have cognitive deficits, such as reduced learning and/or memory. Furthermore, we propose that human FSCN1 should be considered a candidate gene for developmental brain disorders, including mental retardation and autism, especially those for which no obvious neuroanatomical defects are detectable. Hundreds of causative or susceptibility genes for developmental brain disorders remain to be identified (Inlow and Restifo, 2004; Hu-Lince et al., 2005; Owen et al., 2005). The known mental retardation genes include several highly conserved ones involved in actin cytoskeleton structure or regulation, including dystrophin, filaminA, integrin $\alpha-7$, oligophrenin-1, and PAK3 [Inlow and Restifo (2004) and references therein]. Moreover, FSCN1 is an upregulated target of the transcriptional coactivator CREB (cAMP response elementbinding protein) binding protein (Megiorni et al., 2005), mutations of which cause Rubinstein-Taybi mental retardation syndrome (Petrij et al., 1995). Thus, the cognitive deficits in that syndrome may result in part from fascin deficiency during brain development. 


\section{Contribution of computational approaches to quantifying neuron morphology}

Neurite-curvature measurement represents a novel and significant addition to the quantification tools available for cultured neurons, especially because our method provides fully automated analysis of skeletonized images. Our ability to quantify neuronal phenotypes in vitro at single-cell resolution and to classify neuron curvature will facilitate screening for genetic or pharmacological modifiers of the filagree phenotype. This strategy could reveal new genetic pathways underlying the molecular basis of neuronal morphogenesis and its disruption in disease conditions. Moreover, because of the extraordinary phylogenetic conservation of MR genes (Inlow and Restifo, 2004), it could provide a powerful novel drug discovery tool for developmental brain disorders.

\section{References}

Adams JC (2004) Roles of fascin in cell adhesion and motility. Curr Opin Cell Biol 16:590-596.

Arbeitman MN, Fleming AA, Siegal ML, Null BH, Baker BS (2004) A genomic analysis of Drosophila somatic sexual differentiation and its regulation. Development 131:2007-2021.

Arimura N, Kaibuchi K (2005) Key regulators in neuronal polarity. Neuron 48:881-884.

Baas PW (1999) Microtubules and neuronal polarity: lessons from mitosis. Neuron 22:23-31.

Bainbridge SP, Bownes M (1988) Ecdysteroid titers during Drosophila metamorphosis. Insect Biochem 18:185-197.

Bardoni B, Mandel JL, Fisch GS (2000) FMR1 gene and fragile X syndrome. Am J Med Genet 97:153-163.

Bender HA (1960) Studies on the expression of various singed alleles in Drosophila melanogaster. Genetics 45:867-883.

Bier E (2005) Drosophila, the golden bug, emerges as a tool for human genetics. Nat Rev Genet 6:9-23.

Blanpied TA, Ehlers MD (2004) Microanatomy of dendritic spines: emerging principles of synaptic pathology in psychiatric and neurological disease. Biol Psychiatry 55:1121-1127.

Boquet I, Hitier R, Dumas M, Chaminade M, Preat T (2000) Central brain postembryonic development in Drosophila: implication of genes expressed at the interhemispheric junction. J Neurobiol 42:33-48.

Boyd L, O'Toole E, Thummel CS (1991) Patterns of E74A RNA and protein expression at the onset of metmorphosis. Development 112:981-995.

Brand AH, Dormand E-L (1995) The GAL4 system as a tool for unravelling the mysteries of the Drosophila nervous system. Curr Opin Neurobiol 5:572-578.

Briese M, Esmaeili B, Sattelle DB (2005) Is spinal muscular atrophy the result of defects in motor neuron processes? BioEssays 27:946-957.

Bryan J, Edwards R, Matsudaira P, Otto J, Wulfkuhle J (1993) Fascin, an echinoid actin-bundling protein, is a homolog of the Drosophila singed gene product. Proc Natl Acad Sci USA 90:9115-9119.

Cant K, Knowles BA, Mooseker MS, Cooley L (1994) Drosophila singed, a fascin homolog, is required for actin bundle formation during oogenesis and bristle extension. J Cell Biol 125:369-380.

Carolina Biological Supply Company (1988) Carolina Drosophila manual, p 17. Burlington, NC: Carolina Biological Supply.

Christiansen AE, Keisman EL, Ahmad SM, Baker BS (2002) Sex comes in from the cold: the integration of sex and pattern. Trends Genet 18:510-516.

Clark IE, Jan LY, Jan YN (1997) Reciprocal localization of Nod and kinesin fusion proteins indicates microtubule polarity in the Drosophila oocyte, epithelium, neuron and muscle. Development 124:461-470.

Cohan CS, Welnhofer EA, Zhao L, Matsumura F, Yamashiro S (2001) Role of the actin bundling protein fascin in growth cone morphogenesis: localization in filopodia and lamellipodia. Cell Motil Cytoskeleton 48:109-120.

Cohen-Cory S (2002) The developing synapse: construction and modulation of synaptic structures and circuits. Science 298:770-776.

De Arcangelis A, Georges-Labouesse E, Adams JC (2004) Expression of fascin-1, the gene encoding the actin-bundling protein fascin-1, during mouse embryogenesis. Gene Expr Patterns 4:637-643.
De La Cruz E, Pollard TD (1994) Transient kinetic analysis of rhodamine phalloidin binding to actin filaments. Biochemistry 33:14387-14392.

Dillon C, Goda Y (2005) The actin cytoskeleton: integrating form and function at the synapse. Annu Rev Neurosci 28:25-55.

Esch T, Lemmon V, Banker G (2000) Differential effects of NgCAM and $\mathrm{N}$-cadherin on the development of axons and dendrites by cultured hippocampal neurons. J Neurocytol 29:215-223.

Farmer LM, Hagmann J, Dagan D, Matus AI, Levitan IB (1992) Directional control of neurite outgrowth from cultured hippocampal neurons is modulated by the lectin concanavalin A. J Neurobiol 23:354-363.

Ferveur JF, Savarit F, O’Kane CJ, Sureau G, Greenspan RJ, Jallon JM (1997) Genetic feminization of pheromones and its behavioral consequences in Drosophila males. Science 276:1555-1558.

Fletcher JC, Burtis KC, Hogness DS, Thummel CS (1995) The Drosophila E74 gene is required for metamorphosis and plays a role in the polytene chromosome puffing response to ecdysone. Development 121: 1411-1421.

Frambach I, Rössler W, Winkler M, Schürmann F-W (2004) F-actin at identified synapses in the mushroom body neuropil of the insect brain. J Comp Neurol 475:303-314.

Guild GM, Connelly PS, Ruggiero L, Vranich KA, Tilney LG (2003) Long continuous actin bundles in Drosophila bristles are constructed by overlapping short filaments. J Cell Biol 162:1069-1077.

Hastie T, Tibshiriani R, Friedman J (2001) The elements of statistical learning; data mining, inference, and prediction. New York: Springer.

Heisenberg M (2003) Mushroom body memoir: from maps to models. Nat Rev Neurosci 4:266-275.

Hu-Lince D, Craig DW, Huentelman MJ, Stephan DA (2005) The autism genome project: goals and strategies. Am J Pharmacogenomics 5:233-246.

Inlow JK, Restifo LL (2004) Molecular and comparative genetics of mental retardation. Genetics 166:835-881.

Jacobson C, Schnapp B, Banker GA (2006) A change in the selective translocation of the Kinesin-1 motor domain marks the initial specification of the axon. Neuron 49:797-804.

Kalil K, Dent EW (2005) Touch and go: guidance cues signal to the growth cone cytoskeleton. Curr Opin Neurobiol 15:521-526.

Kaufmann WE, Moser HW (2000) Dendritic anomalies in disorders associated with mental retardation. Cereb Cortex 10:981-991.

Kido A, Ito K (2002) Mushroom bodies are not required for courtship behavior by normal and sexually mosaic Drosophila. J Neurobiol 52:302-311.

Kim YT, Wu C-F (1996) Reduced growth cone motility in cultured neurons from Drosophila memory mutants with a defective cAMP cascade. J Neurosci 16:5593-5602.

Kraft R, Levine RB, Restifo LL (1998) The steroid hormone 20hydroxyecdysone enhances neurite growth of Drosophila mushroom body neurons isolated during metamorphosis. J Neurosci 18:8886-8899.

Kureishy N, Sapountzi V, Prag S, Anilkumar N, Adams JC (2002) Fascins, and their roles in cell structure and function. BioEssays 24:350-361.

Lee G, Hall JC, Park JH (2002) Doublesex gene expression in the central nervous system of Drosophila melanogaster. J Neurogenet 16:229-248.

Lee SJ, Romeo RD, Svenningsson P, Campomanes CR, Allen PB, Greengard P, McEwen BS (2004) Estradiol affects spinophilin protein differently in gonadectomized males and females. Neuroscience 127:983-988.

Lee T, Lee A, Luo L (1999) Development of the Drosophila mushroom bodies: sequential generation of three distinct types of neurons from a neuroblast. Development 126:4065-4076.

Lee T, Marticke S, Sung C, Robinow S, Luo L (2000a) Cell-autonomous requirement of the USP/EcR-B ecdysone receptor for mushroom body neuronal remodeling in Drosophila. Neuron 28:807-818.

Lee T, Winter C, Marticke SS, Lee A, Luo L (2000b) Essential roles of Drosophila RhoA in the regulation of neuroblast proliferation and dendritic but not axonal morphogenesis. Neuron 25:307-316.

Lindsley TA, Kerlin AM, Rising LJ (2003) Time-lapse analysis of ethanol's effects on axon growth in vitro. Dev Brain Res 147:191-199.

Luo L (2002) Actin cytoskeleton regulation in neuronal morphogenesis and structural plasticity. Annu Rev Cell Dev Biol 18:601-635.

Manoli DS, Foss M, Villella A, Taylor BJ, Hall JC, Baker BS (2005) Malespecific fruitless specifies the neural substrates of Drosophila courtship behaviour. Nature 436:395-400.

Megiorni F, Indovina P, Mora B, Mazzilli MC (2005) Minor expression of 
fascin-1 gene (FSCN1) in NTera2 cells depleted of CREB-binding protein. Neurosci Lett 381:169-174.

Michel CI, Kraft R, Restifo LL (2004) Defective neuronal development in the mushroom bodies of Drosophila fragile $X$ mental retardation $1 \mathrm{mu}-$ tants. J Neurosci 24:5798-5809.

Miranda P, Williams CL, Einstein G (1999) Granule cells in aging rats are sexually dimorphic in their response to estradiol. J Neurosci 19:3316-3325.

Myers JL, Well AD (1991) Research design and statistical analysis. New York: HarperCollins.

Nassogne MC, Evrard P, Courtoy PJ (1995) Selective neuronal toxicity of cocaine in embryonic mouse brain cocultures. Proc Natl Acad Sci USA 92:11029-11033.

O’Dell KM, Armstrong JD, Yang MY, Kaiser K (1995) Functional dissection of the Drosophila mushroom bodies by selective feminization of genetically defined subcompartments. Neuron 15:55-61.

Ouyang Y, Wong M, Capani F, Rensing N, Lee CS, Liu Q, Neusch C, Martone ME, Wu JY, Yamada K, Ellisman MH, Choi DW (2005) Transient decrease in F-actin may be necessary for translocation of proteins into dendritic spines. Eur J Neurosci 22:2995-3005.

Owen MJ, Craddock N, O’Donovan MC (2005) Schizophrenia: genes at last? Trends Genet 21:518-525.

Paterson J, O'Hare K (1991) Structure and transcription of the singed locus of Drosophila melanogaster. Genetics 129:1073-1084.

Peraud A, Mondal S, Hawkins C, Mastronardi M, Bailey K, Rutka JT (2003) Expression of fascin, an actin-bundling protein, in astrocytomas of varying grades. Brain Tumor Pathol 20:53-58.

Petrij F, Giles RH, Dauwerse HG, Saris JJ, Hennekam RC, Masuno M, Tommerup N, van Ommen GJ, Goodman RH, Peters DJ, Breuning MH (1995) Rubinstein-Taybi syndrome caused by mutations in the transcriptional co-activator CBP. Nature 376:348-351.

Restifo LL (2005) Mental retardation genes in Drosophila: new approaches to understanding and treating developmental brain disorders. Ment Retard Dev Disabil Res Rev 11:286-294.

Restifo LL, White K (1991) Mutations in a steroid hormone-regulated gene disrupt the metamorphosis of the central nervous system in Drosophila. Dev Biol 148:174-194.

Richards G (1981) The radioimmunoassay of ecdysteroid titers in Drosophila melanogaster. Mol Cell Endocrinol 21:181-197.

Robinson TE, Kolb B (2004) Structural plasticity associated with exposure to drugs of abuse. Neuropharmacology 47 [Suppl 1]:33-46.

Rohrbough J, O'Dowd DK, Baines RA, Broadie K (2003) Cellular bases of behavioral plasticity: establishing and modifying synaptic circuits in the Drosophila genetic system. J Neurobiol 54:254-271.

Roma AA, Prayson RA (2005) Fascin expression in 90 patients with glioblastoma multiforme. Ann Diagn Pathol 9:307-311.
Romijn HJ, Mud MT, Wolters PS, Corner MA (1980) Neurite formation in dissociated cerebral cortex in vitro: evidence for clockwise outgrowth and autotopic contacts. Brain Res 192:575-580.

Sanchez-Soriano N, Bottenberg W, Fiala A, Haessler U, Kerassoviti A, Knust E, Lohr R, Prokop A (2005) Are dendrites in Drosophila homologous to vertebrate dendrites? Dev Biol 288:126-138.

Sasaki Y, Hayashi K, Shirao T, Ishikawa R, Kohama K (1996) Inhibition by drebrin of the actin-bundling activity of brain fascin, a protein localized in filopodia of growth cones. J Neurochem 66:980-988.

Schulz RA, Chromey C, Lu M-F, Zhao B, Olson EN (1996) Expression of the D-MEF2 transcription factor in the Drosophila brain suggests a role in neuronal cell differentiation. Oncogene 12:1827-1831.

Strausfeld NJ, Meinertzhagen IA (1998) The insect neuron: types, morphologies, fine structure, and relationship to the architectonics of the insect nervous system. In: Microscopic anatomy of invertebrates, Vol 11B, Insecta (Harrison FW, Locke M, eds), pp 487-538. New York: Wiley.

Sutherland GR (1983) The fragile X chromosome. Int Rev Cytol 81:107-143.

Technau GM (1984) Fiber number in the mushroom bodies of adult Drosophila melanogaster depends on age, sex and experience. J Neurogenet 1:113-126.

Thummel CS (1996) Flies on steroids-Drosophila metamorphosis and the mechanisms of steroid hormone action. Trends Genet 12:306-310.

Tilney LG, Tilney MS, Guild GM (1995) F actin bundles in Drosophila bristles. I. Two filament cross-links are involved in bundling. J Cell Biol 130:629-638.

Tilney LG, Connelly PS, Ruggiero L, Vranich KA, Guild GM, DeRosier D (2004) The role actin filaments play in providing the characteristic curved form of Drosophila bristles. Mol Biol Cell 15:5481-5491.

Tilney LG, DeRosier DJ (2005) How to make a curved Drosophila bristle using straight actin bundles. Proc Natl Acad Sci USA 102:18785-18792.

Victor M, Ropper A (2001) Degenerative diseases of the nervous system. In: Adams and Victor's principles of neurology, Ed 7 (Victor M, Ropper A, eds), pp 1106-1174. San Francisco: McGraw Hill.

Wada Y, Abe T, Takeshita T, Sato H, Yanashima K, Tamai M (2001) Mutation of human retinal fascin gene (FSCN2) causes autosomal dominant retinitis pigmentosa. Invest Ophthalmol Vis Sci 42:2395-2400.

Wada Y, Abe T, Itabashi T, Sato H, Kawamura M, Tamai M (2003) Autosomal dominant macular degeneration associated with 208delG mutation in the FSCN2 gene. Arch Ophthalmol 121:1613-1620.

Yang MY, Armstrong JD, Vilinsky I, Strausfeld NJ, Kaiser K (1995) Subdivision of the Drosophila mushroom bodies by enhancer-trap expression patterns. Neuron 15:45-54.

Zarnescu DC, Shan G, Warren ST, Jin P (2005) Come FLY with us: toward understanding fragile X syndrome. Genes Brain Behav 4:385-392. 